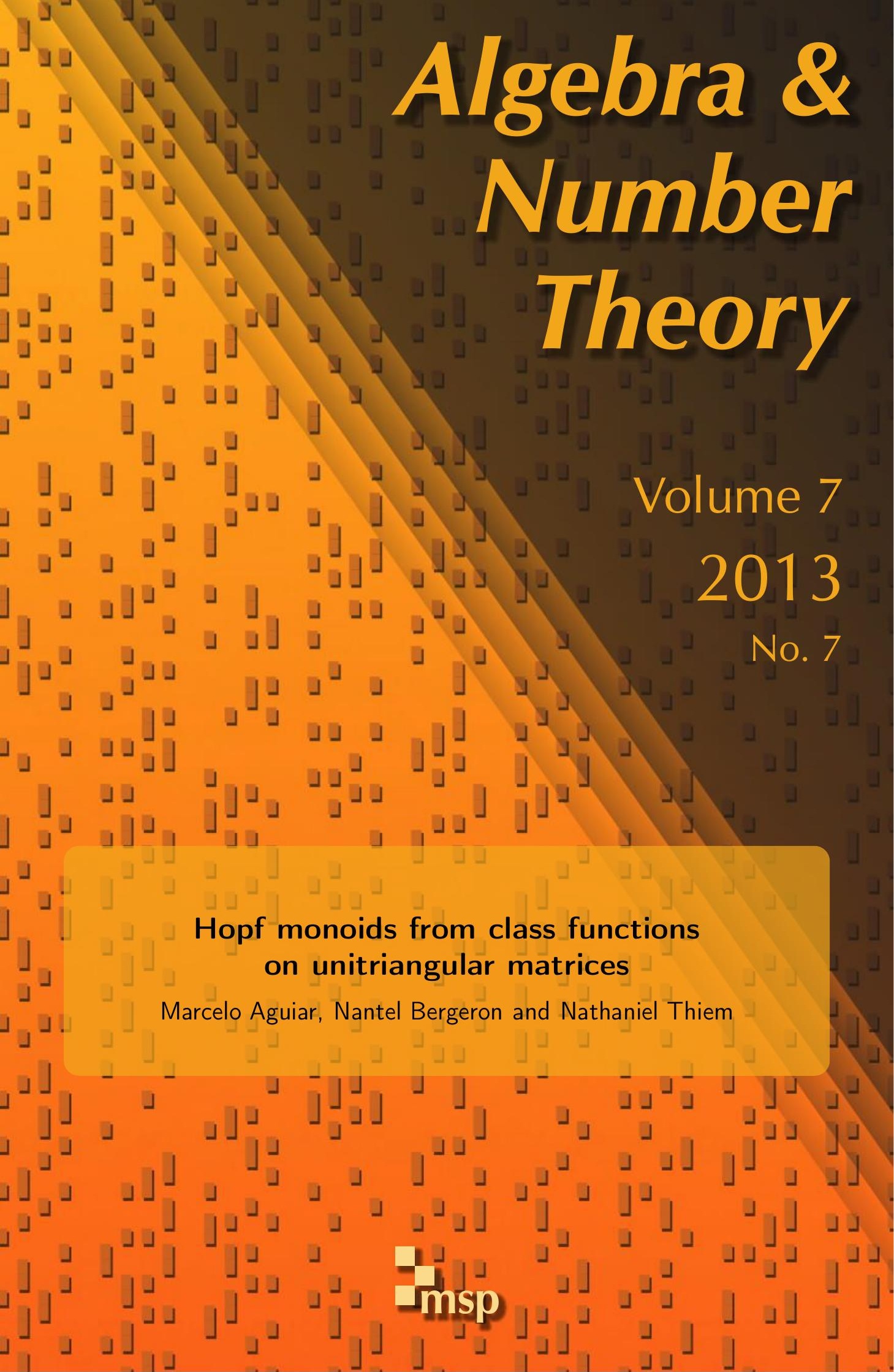




\title{
Hopf monoids from class functions on unitriangular matrices
}

\author{
Marcelo Aguiar, Nantel Bergeron and Nathaniel Thiem
}

\begin{abstract}
We build, from the collection of all groups of unitriangular matrices, Hopf monoids in Joyal's category of species. Such structure is carried by the collection of class function spaces on those groups and also by the collection of superclass function spaces in the sense of Diaconis and Isaacs. Superclasses of unitriangular matrices admit a simple description from which we deduce a combinatorial model for the Hopf monoid of superclass functions in terms of the Hadamard product of the Hopf monoids of linear orders and of set partitions. This implies a recent result relating the Hopf algebra of superclass functions on unitriangular matrices to symmetric functions in noncommuting variables. We determine the algebraic structure of the Hopf monoid: it is a free monoid in species with the canonical Hopf structure. As an application, we derive certain estimates on the number of conjugacy classes of unitriangular matrices.
\end{abstract}

\section{Introduction}

A Hopf monoid (in Joyal's category of species) is an algebraic structure akin to that of a Hopf algebra. Combinatorial structures that compose and decompose give rise to Hopf monoids. These objects are the subject of [Aguiar and Mahajan 2010, Part II]. The few basic notions and examples needed for our purposes are reviewed in Section 1, including the Hopf monoids of linear orders, set partitions, and simple graphs and the Hadamard product of Hopf monoids.

The main goal of this paper is to construct a Hopf monoid out of the groups of unitriangular matrices with entries in a finite field and to do this in a transparent manner. The structure exists on the collection of function spaces on these groups and also on the collections of class function and superclass function spaces. It is induced by two simple operations on this collection of groups: the passage from a

Aguiar is supported in part by NSF grant DMS-1001935. Bergeron is supported in part by CRC and NSERC. Thiem is supported in part by NSF FRG DMS-0854893.

MSC2010: primary 05E10; secondary 05E05, 05E15, 16T05, 16T30, 18D35, 20C33.

Keywords: unitriangular matrix, class function, superclass function, Hopf monoid, Hopf algebra. 
matrix to its principal minors gives rise to the product, and direct sum of matrices gives rise to the coproduct.

Class functions are defined for arbitrary groups. An abstract notion and theory of superclass functions (and supercharacters) for arbitrary groups exists [Diaconis and Isaacs 2008]. While a given group may admit several such theories, there is a canonical choice of superclasses for a special class of groups known as algebra groups. These notions are briefly discussed in Section 4.1. Unitriangular groups are the prototype of such groups, and we employ the corresponding notion of superclasses in Section 4.2. The study of unitriangular superclasses and supercharacters was initiated in [André 1995a; 1995b], making use of the method of Kirillov [1995], and by more elementary means in [Yan 2001].

Preliminaries on unitriangular matrices are discussed in Section 2. The Hopf monoids $\mathbf{f}(\mathrm{U})$ of functions and $\mathbf{c f}(\mathrm{U})$ of class functions are constructed in Section 3. The nature of the construction is fairly general; in particular, the same procedure yields the Hopf monoid $\operatorname{scf}(\mathrm{U})$ of superclass functions in Section 4.2.

Unitriangular matrices over $\mathbb{F}_{2}$ may be identified with simple graphs, and direct sums and the passage to principal minors correspond to simple operations on graphs. This yields a combinatorial model for $f(U)$ in terms of the Hadamard product of the Hopf monoids of linear orders and of graphs, as discussed in Section 3.6. The conjugacy classes on the unitriangular groups exhibit great complexity and considerable attention has been devoted to their study [Goodwin 2006; Higman 1960; Kirillov 1995; Vera-López et al. 2008]. We do not attempt an explicit combinatorial description of the Hopf monoid $\mathrm{cf}(\mathrm{U})$. On the other hand, superclasses are wellunderstood (Section 4.3), and such a combinatorial description exists for $\operatorname{scf}(\mathrm{U})$. In Section 4.5, we obtain a combinatorial model in terms of the Hadamard product of the Hopf monoids of linear orders and of set partitions. This has as a consequence the main result of [Aguiar et al. 2012], as we explain in Section 6.2.

Employing the combinatorial models, we derive structure theorems for the Hopf monoids $f(U)$ and $\operatorname{scf}(U)$ in Section 5. Our main results state that both are free monoids with the canonical Hopf structure (in which the generators are primitive).

Applications are presented in Section 6. With the aid of Lagrange's theorem for Hopf monoids, one may derive estimates on the number of conjugacy classes of unitriangular matrices in the form of certain recursive inequalities. We obtain this application in Section 6.1, where we also formulate a refinement of Higman's conjecture on the polynomiality of these numbers. Other applications involving the Hopf algebra of superclass functions of [Aguiar et al. 2012] are given in Section 6.2.

We employ two fields: the base field $\mathbb{k}$ and the field of matrix entries $\mathbb{F}$. We consider algebras and groups of matrices with entries in $\mathbb{F}$; all other vector spaces are over $\mathbb{k}$. The field of matrix entries is often assumed to be finite and sometimes to be $\mathbb{F}_{2}$. 


\section{Hopf monoids}

We review the basics on Hopf monoids and recall three examples built from linear orders, set partitions, and simple graphs, respectively. We also consider the Hadamard product of Hopf monoids. In later sections, Hopf monoids are built from functions on unitriangular matrices. The constructions of this section will allow us to provide combinatorial models for them.

1.1. Species and Hopf monoids. For the precise definitions of vector species and Hopf monoid, we refer to [Aguiar and Mahajan 2010, Chapter 8]. The main ingredients are reviewed below.

A vector species $\boldsymbol{p}$ is a collection of vector spaces $\boldsymbol{p}[I]$, one for each finite set $I$, equivariant with respect to bijections $I \cong J$. A morphism of species $f: \boldsymbol{p} \rightarrow \boldsymbol{q}$ is a collection of linear maps $f_{I}: \boldsymbol{p}[I] \rightarrow \boldsymbol{q}[I]$ that commute with bijections.

A decomposition of a finite set $I$ is a finite sequence $\left(S_{1}, \ldots, S_{k}\right)$ of disjoint subsets of $I$ whose union is $I$. In this situation, we write

$$
I=S_{1} \sqcup \cdots \sqcup S_{k} .
$$

A Hopf monoid consists of a vector species $\boldsymbol{h}$ equipped with two collections $\mu$ and $\Delta$ of linear maps

$$
\boldsymbol{h}\left[S_{1}\right] \otimes \boldsymbol{h}\left[S_{2}\right] \stackrel{\mu_{S_{1}, S_{2}}}{\longrightarrow} \boldsymbol{h}[I] \quad \text { and } \quad \boldsymbol{h}[I] \stackrel{\Delta_{S_{1}, S_{2}}}{\longrightarrow} \boldsymbol{h}\left[S_{1}\right] \otimes \boldsymbol{h}\left[S_{2}\right] .
$$

There is one map in each collection for each finite set $I$ and each decomposition $I=S_{1} \sqcup S_{2}$. This data is subject to a number of axioms, of which the main ones follow.

Associativity. For each decomposition $I=S_{1} \sqcup S_{2} \sqcup S_{3}$, the diagrams

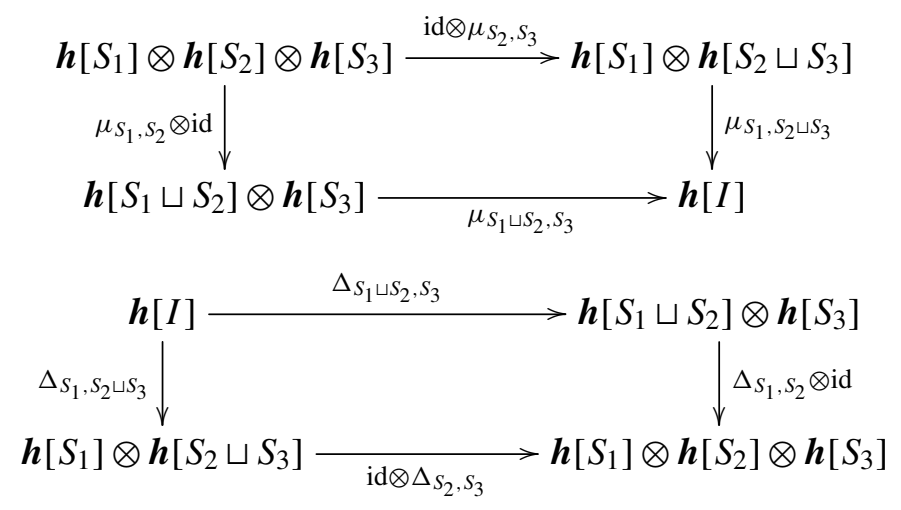

commute. 
Compatibility. Fix decompositions $S_{1} \sqcup S_{2}=I=T_{1} \sqcup T_{2}$, and consider the resulting pairwise intersections

$$
A:=S_{1} \cap T_{1}, \quad B:=S_{1} \cap T_{2}, \quad C:=S_{2} \cap T_{1}, \quad \text { and } \quad D:=S_{2} \cap T_{2},
$$

as illustrated below:
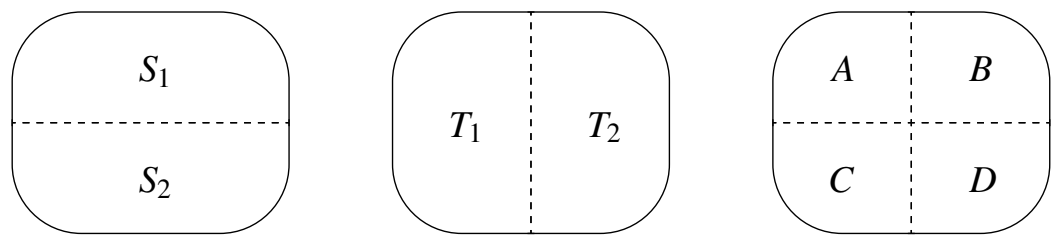

For any such pair of decompositions, the diagram

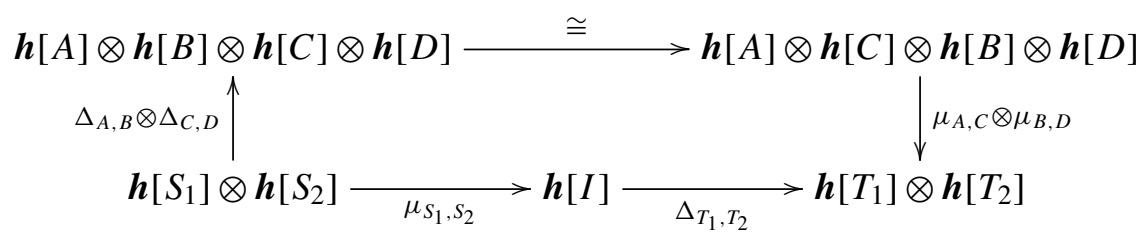

must commute. The top arrow stands for the map that interchanges the middle factors.

In addition, the Hopf monoid $\boldsymbol{h}$ is connected if $\boldsymbol{h}[\varnothing]=\mathbb{k}$ and the maps

$$
\boldsymbol{h}[I] \otimes \boldsymbol{h}[\varnothing] \underset{\Delta_{I, \varnothing}}{\stackrel{\mu_{I, \varnothing}}{\rightleftarrows}} \boldsymbol{h}[I] \quad \text { and } \quad \boldsymbol{h}[\varnothing] \otimes \boldsymbol{h}[I] \underset{\Delta_{\varnothing, I}}{\stackrel{\mu_{\varnothing, I}}{\rightleftarrows}} \boldsymbol{h}[I]
$$

are the canonical identifications.

The collection $\mu$ is the product, and the collection $\Delta$ is the coproduct of the Hopf monoid $\boldsymbol{h}$.

A Hopf monoid is (co)commutative if the left (right) diagram below commutes for all decompositions $I=S_{1} \sqcup S_{2}$ :
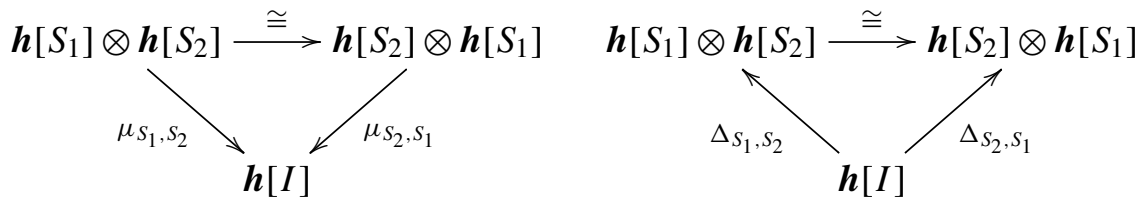

The top arrows stand for the map that interchanges the factors.

A morphism of Hopf monoids $f: \boldsymbol{h} \rightarrow \boldsymbol{k}$ is a morphism of species that commutes with $\mu$ and $\Delta$. 
1.2. The Hopf monoid of linear orders. For any finite set $I, \mathrm{~L}[I]$ is the set of all linear orders on $I$. For instance, if $I=\{a, b, c\}$,

$$
\mathrm{L}[I]=\{a b c, b a c, a c b, b c a, c a b, c b a\} .
$$

Let $\boldsymbol{L}[I]$ denote the vector space with basis $L[I]$. The collection $\boldsymbol{L}$ is a vector species.

Let $I=S_{1} \sqcup S_{2}$. Given linear orders $\ell_{i}$ on $S_{i}, i=1,2$, their concatenation $\ell_{1} \cdot \ell_{2}$ is a linear order on $I$. This is the list consisting of the elements of $S_{1}$ as ordered by $\ell_{1}$ followed by those of $S_{2}$ as ordered by $\ell_{2}$. Given a linear order $\ell$ on $I$ and $S \subseteq I$, the restriction $\left.\ell\right|_{S}$ (the list consisting of the elements of $S$ written in the order in which they appear in $\ell$ ) is a linear order on $S$. These operations give rise to maps

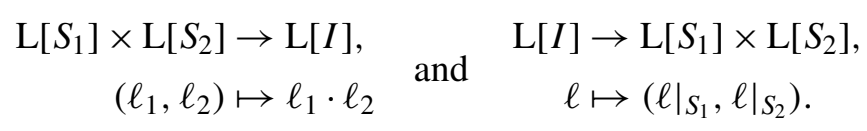

Extending by linearity, we obtain linear maps

$$
\mu_{S_{1}, S_{2}}: \boldsymbol{L}\left[S_{1}\right] \otimes \boldsymbol{L}\left[S_{2}\right] \rightarrow \boldsymbol{L}[I] \quad \text { and } \quad \Delta_{S_{1}, S_{2}}: \boldsymbol{L}[I] \rightarrow \boldsymbol{L}\left[S_{1}\right] \otimes \boldsymbol{L}\left[S_{2}\right]
$$

that turn $\boldsymbol{L}$ into a Hopf monoid. For instance, given linear orders $\ell_{i}$ on $S_{i}, i=1,2$, the commutativity of (4) boils down to the fact that the concatenation of $\left.\ell_{1}\right|_{A}$ and $\left.\ell_{2}\right|_{C}$ agrees with the restriction to $T_{1}$ of $\ell_{1} \cdot \ell_{2}$. The Hopf monoid $\boldsymbol{L}$ is cocommutative but not commutative. For more details, see [Aguiar and Mahajan 2010, Section 8.5].

1.3. The Hopf monoid of set partitions. A partition of a finite set $I$ is a collection $X$ of disjoint nonempty subsets whose union is $I$. The subsets are the blocks of $X$.

Given a partition $X$ of $I$ and $S \subseteq I$, the restriction $\left.X\right|_{S}$ is the partition of $S$ whose blocks are the nonempty intersections of the blocks of $X$ with $S$. Let $I=S_{1} \sqcup S_{2}$. Given partitions $X_{i}$ of $S_{i}, i=1,2$, their union is the partition $X_{1} \sqcup X_{2}$ of $I$ whose blocks are the blocks of $X_{1}$ and the blocks of $X_{2}$. A quasishuffle of $X_{1}$ and $X_{2}$ is any partition of $I$ whose restriction to $S_{i}$ is $X_{i}, i=1,2$.

Let $\Pi[I]$ denote the set of partitions of $I$ and $\Pi[I]$ the vector space with basis $\Pi[I]$. A Hopf monoid structure on $\Pi$ is defined and studied in [Aguiar and Mahajan 2010, Section 12.6]. Among its various linear bases, we are interested in the basis $\left\{m_{X}\right\}$ on which the operations are as follows. The product

$$
\mu_{S_{1}, S_{2}}: \Pi\left[S_{1}\right] \otimes \Pi\left[S_{2}\right] \rightarrow \Pi[I]
$$

is given by

$$
\mu_{S_{1}, S_{2}}\left(m_{X_{1}} \otimes m_{X_{2}}\right)=\sum_{\substack{\left.X\right|_{S_{1}}=\left.X_{1} \\ X\right|_{S_{2}}=X_{2}}} m_{X}
$$


The coproduct

$$
\Delta_{S_{1}, S_{2}}: \Pi[I] \rightarrow \Pi\left[S_{1}\right] \otimes \Pi\left[S_{2}\right]
$$

is given by

$$
\Delta_{S_{1}, S_{2}}\left(m_{X}\right)= \begin{cases}m_{\left.X\right|_{1}} \otimes m_{\left.X\right|_{S_{2}}} & \text { if } S_{1} \text { is the union of some blocks of } X, \\ 0 & \text { otherwise. }\end{cases}
$$

Note that the following conditions are equivalent for a partition $X$ of $I$ :

- $S_{1}$ is the union of some blocks of $X$.

- $S_{2}$ is the union of some blocks of $X$.

- $X=\left.\left.X\right|_{S_{1}} \sqcup X\right|_{S_{2}}$.

These operations turn the species $\Pi$ into a Hopf monoid that is both commutative and cocommutative.

1.4. The Hopf monoid of simple graphs. A (simple) graph $g$ on a finite set $I$ is a relation on $I$ that is symmetric and irreflexive. The elements of $I$ are the vertices of $g$ There is an edge between two vertices when they are related by $g$.

Given a graph $g$ on $I$ and $S \subseteq I$, the restriction $\left.g\right|_{S}$ is the graph on $S$ whose edges are the edges of $g$ between elements of $S$. Let $I=S_{1} \sqcup S_{2}$. Given graphs $g_{i}$ of $S_{i}, i=1,2$, their union is the graph $g_{1} \sqcup g_{2}$ of $I$ whose edges are those of $g_{1}$ and those of $g_{2}$. A quasishuffle of $g_{1}$ and $g_{2}$ is any graph on $I$ whose restriction to $S_{i}$ is $g_{i}, i=1,2$.

Let $\mathrm{G}[I]$ denote the set of graphs on $I$ and $\boldsymbol{G}[I]$ the vector space with basis $\mathrm{G}[I]$. A Hopf monoid structure on $\boldsymbol{G}$ is defined and studied in [Aguiar and Mahajan 2010, Section 13.2]. We are interested in the basis $\left\{m_{g}\right\}$ on which the operations are as follows. The product

$$
\mu_{S_{1}, S_{2}}: \boldsymbol{G}\left[S_{1}\right] \otimes \boldsymbol{G}\left[S_{2}\right] \rightarrow \boldsymbol{G}[I]
$$

is given by

$$
\mu_{S_{1}, S_{2}}\left(m_{g_{1}} \otimes m_{g_{2}}\right)=\sum_{\substack{\left.g\right|_{S_{1}}=\left.g_{1} \\ g\right|_{S_{2}}=g_{2}}} m_{g} .
$$

The coproduct

$$
\Delta_{S_{1}, S_{2}}: \boldsymbol{G}[I] \rightarrow \boldsymbol{G}\left[S_{1}\right] \otimes \boldsymbol{G}\left[S_{2}\right]
$$

is given by

$$
\Delta_{S_{1}, S_{2}}\left(m_{g}\right)= \begin{cases}m_{g \mid S_{1}} \otimes m_{g \mid S_{2}} & \text { if no edge of } g \text { connects } S_{1} \text { to } S_{2}, \\ 0 & \text { otherwise. }\end{cases}
$$

Note that no edge of $g$ connects $S_{1}$ to $S_{2}$ if and only if $g=\left.\left.g\right|_{S_{1}} \sqcup g\right|_{S_{2}}$. 
These operations turn the species $\boldsymbol{G}$ into a Hopf monoid that is both commutative and cocommutative.

Remark 1. The dual of a species $\boldsymbol{p}$ is the collection $\boldsymbol{p}^{*}$ of dual vector spaces: $\boldsymbol{p}^{*}[I]=\boldsymbol{p}[I]^{*}$. A species $\boldsymbol{p}$ is said to be finite-dimensional if each space $\boldsymbol{p}[I]$ is finite-dimensional. Dualizing the operations of a finite-dimensional Hopf monoid $\boldsymbol{h}$, one obtains a Hopf monoid $\boldsymbol{h}^{*}$. The Hopf monoid $\boldsymbol{h}$ is called self-dual if $\boldsymbol{h} \cong \boldsymbol{h}^{*}$. In general, such isomorphism is not unique.

Over a field of characteristic 0, a Hopf monoid that is connected, commutative, and cocommutative is always self-dual. This is a consequence of the Cartier-MilnorMoore theorem. (The isomorphism with the dual is not canonical.)

In particular, the Hopf monoids $\boldsymbol{\Pi}$ and $\boldsymbol{G}$ are self-dual. In [Aguiar and Mahajan 2010], the preceding descriptions of these Hopf monoids are stated in terms of their duals $\boldsymbol{\Pi}^{*}$ and $\boldsymbol{G}^{*}$. A different description of $\boldsymbol{\Pi}$ is given in [Aguiar and Mahajan 2010, Section 12.6.2]. To reconcile the two, one should use the explicit isomorphism $\Pi \cong \Pi^{*}$ given in [Aguiar and Mahajan 2010, Proposition 12.48].

1.5. The Hadamard product. Given species $\boldsymbol{p}$ and $\boldsymbol{q}$, their Hadamard product is the species $\boldsymbol{p} \times \boldsymbol{q}$ defined by

$$
(\boldsymbol{p} \times \boldsymbol{q})[I]=p[I] \otimes \boldsymbol{q}[I] .
$$

If $\boldsymbol{h}$ and $\boldsymbol{k}$ are Hopf monoids, then so is $\boldsymbol{h} \times \boldsymbol{k}$ with the following operations. Let $I=S_{1} \sqcup S_{2}$. The product is

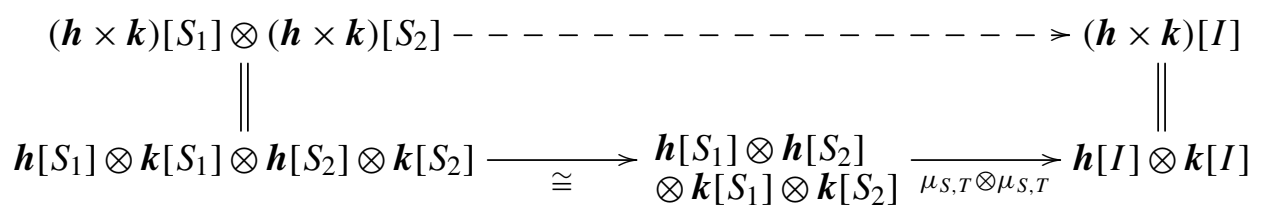

and the coproduct is defined dually. If $\boldsymbol{h}$ and $\boldsymbol{k}$ are (co)commutative, then so is $\boldsymbol{h} \times \boldsymbol{k}$. For more details, see [Aguiar and Mahajan 2010, Section 8.13].

\section{Unitriangular matrices}

This section sets up the basic notation pertaining to unitriangular matrices and discusses two simple but important constructions: direct sum of matrices and the passage from a matrix to its principal minors. The Hopf monoid constructions of later sections are based on them. The key results are Lemmas 2 and 3. The former is the reason why we must use unitriangular matrices: for arbitrary matrices, the passage to principal minors is not multiplicative. The latter will be responsible (in later sections) for the necessary compatibility between the product and coproduct of the Hopf monoids. 
Let $\mathbb{F}$ be a field, $I$ a finite set, and $\ell$ a linear order on $I$. Let $\mathrm{M}(I)$ denote the algebra of matrices

$$
A=\left(a_{i j}\right)_{i, j \in I}, \quad a_{i j} \in \mathbb{F} \text { for all } i, j \in I .
$$

The general linear group GL(I) consists of the invertible matrices in $\mathrm{M}(I)$, and the subgroup $\mathrm{U}(I, \ell)$ consists of the upper $\ell$-unitriangular matrices

$$
U=\left(u_{i j}\right)_{i, j \in I}, \quad u_{i i}=1 \text { for all } i \in I \text { and } u_{i j}=0 \text { whenever } i>_{\ell} j .
$$

If $\ell^{\prime}$ is another linear order on $I$, then $\mathrm{U}(I, \ell)$ and $\mathrm{U}\left(I, \ell^{\prime}\right)$ are conjugate subgroups of GL(I). However, we want to keep track of all groups in this collection and of the manner in which they interact.

2.1. Direct sum of matrices. Suppose $I=S_{1} \sqcup S_{2}$ is a decomposition. Given $A=\left(a_{i j}\right) \in \mathrm{M}\left(S_{1}\right)$ and $B=\left(b_{i j}\right) \in \mathrm{M}\left(S_{2}\right)$, their direct sum is

$$
A \oplus B=\left(c_{i j}\right) \in \mathrm{M}(I)
$$

the matrix with entries

$$
c_{i j}= \begin{cases}a_{i j} & \text { if both } i, j \in S_{1} \\ b_{i j} & \text { if both } i, j \in S_{2} \\ 0 & \text { otherwise }\end{cases}
$$

Let $\ell \in \mathrm{L}[I]$. The direct sum of an $\left.\ell\right|_{S_{1}}$-unitriangular and an $\left.\ell\right|_{S_{2}}$-unitriangular matrix is $\ell$-unitriangular. The morphism of algebras

$$
\mathrm{M}\left(S_{1}\right) \times \mathrm{M}\left(S_{2}\right) \rightarrow \mathrm{M}(I), \quad(A, B) \mapsto A \oplus B
$$

thus restricts to a morphism of groups

$$
\sigma_{S_{1}, S_{2}}: \mathrm{U}\left(S_{1},\left.\ell\right|_{S_{1}}\right) \times \mathrm{U}\left(S_{2},\left.\ell\right|_{S_{2}}\right) \rightarrow \mathrm{U}(I, \ell) .
$$

(The dependence of $\sigma_{S_{1}, S_{2}}$ on $\ell$ is left implicit.)

Direct sum of matrices is associative; thus, for any decomposition $I=S_{1} \sqcup S_{2} \sqcup S_{3}$, the diagram

$$
\begin{gathered}
\mathrm{U}\left(S_{1},\left.\ell\right|_{S_{1}}\right) \times \mathrm{U}\left(S_{2},\left.\ell\right|_{S_{2}}\right) \times \mathrm{U}\left(S_{3},\left.\ell\right|_{S_{3}}\right) \stackrel{\sigma_{S_{1}, S_{2}} \times \mathrm{id}}{\longrightarrow} \mathrm{U}\left(S_{1} \sqcup S_{2},\left.\ell\right|_{S_{1} \sqcup S_{2}}\right) \times \mathrm{U}\left(S_{3},\left.\ell\right|_{S_{3}}\right) \\
\quad{ }_{\mathrm{id} \times \sigma_{S_{2}, S_{3}}} \downarrow \\
\mathrm{U}\left(S_{1},\left.\ell\right|_{S_{1}}\right) \times \mathrm{U}\left(S_{2} \sqcup S_{3},\left.\ell\right|_{S_{2} \sqcup S_{3}}\right) \stackrel{\sigma_{S_{1} \sqcup S_{2}, S_{3}}}{\longrightarrow} \mathrm{U}(I, \ell)
\end{gathered}
$$


commutes. Note also that, with these definitions, $A \oplus B$ and $B \oplus A$ are the same matrix. Thus, the following diagram commutes:

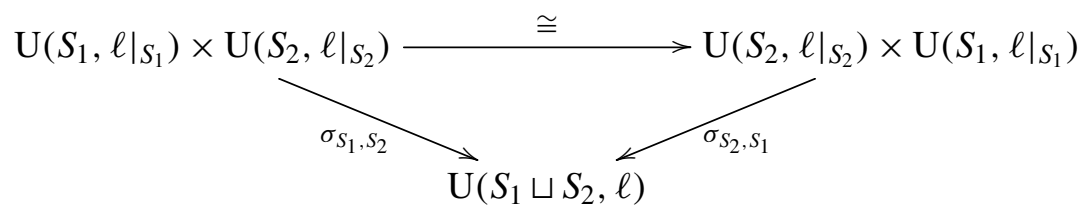

2.2. Principal minors. Given $A=\left(a_{i j}\right) \in \mathrm{M}(I)$, the principal minor indexed by $S \subseteq I$ is the matrix

$$
A_{S}=\left(a_{i j}\right)_{i, j \in S}
$$

In general, $A_{S}$ is not invertible even if $A$ is. In addition, the assignment $A \mapsto A_{S}$ does not preserve multiplications. On the other hand, if $U$ is $\ell$-unitriangular, then $U_{S}$ is $\left.\ell\right|_{S}$-unitriangular. In regard to multiplicativity, we have the following basic fact.

We say that $S$ is an $\ell$-segment if $i, k \in S$ and $i<_{\ell} j<_{\ell} k$ imply that also $j \in S$.

Let $E_{i j} \in \mathrm{M}(I)$ denote the elementary matrix in which the $(i, j)$ entry is 1 and all other entries are 0 .

Lemma 2. Let $\ell \in \mathrm{L}[I]$ and $S \subseteq I$. The map

$$
\mathrm{U}(I, \ell) \rightarrow \mathrm{U}\left(S,\left.\ell\right|_{S}\right), \quad U \mapsto U_{S}
$$

is a morphism of groups if and only if $S$ is an $\ell$-segment.

Proof. Suppose the map is a morphism of groups. Let $i, j, k \in I$ be such that $i, k \in S$ and $i<_{\ell} j<_{\ell} k$. The matrices

$$
\mathrm{Id}+E_{i j} \quad \text { and } \quad \mathrm{Id}+E_{j k}
$$

are in $\mathrm{U}(I, \ell)$, and

$$
\left(\mathrm{Id}+E_{i j}\right) \cdot\left(\mathrm{Id}+E_{j k}\right)=\mathrm{Id}+E_{i j}+E_{j k}+E_{i k} .
$$

If $j \notin S$, then the two matrices are in the kernel of the map while their product is mapped to Id $+E_{i k} \neq \mathrm{Id}$. Thus, $j \in S$ and $S$ is an $\ell$-segment.

The converse implication follows from the fact that if $U$ and $V$ are $\ell$-unitriangular, then the $(i, k)$ entry of $U V$ is

$$
\sum_{i \leq \ell j \leq \ell k} u_{i j} v_{j k} .
$$

Let $I=S_{1} \sqcup S_{2}$ be a decomposition with $\ell_{i} \in \mathrm{L}\left[S_{i}\right], i=1$, 2. We define a map

$$
\pi_{S_{1}, S_{2}}: \mathrm{U}\left(I, \ell_{1} \cdot \ell_{2}\right) \rightarrow \mathrm{U}\left(S_{1}, \ell_{1}\right) \times \mathrm{U}\left(S_{2}, \ell_{2}\right)
$$

by

$$
U \mapsto\left(U_{S_{1}}, U_{S_{2}}\right)
$$


Note that $S_{1}$ is an initial segment for $\ell_{1} \cdot \ell_{2}$ and $S_{2}$ is a final segment for $\ell_{1} \cdot \ell_{2}$. Thus, $\pi_{S_{1}, S_{2}}$ is a morphism of groups by Lemma 2 .

If $R \subseteq S \subseteq I$, then $\left(A_{S}\right)_{R}=A_{R}$. This implies the following commutativity for any decomposition $I=S_{1} \sqcup S_{2} \sqcup S_{3}$ and $\ell_{i} \in \mathrm{L}\left[S_{i}\right], i=1,2,3$ :

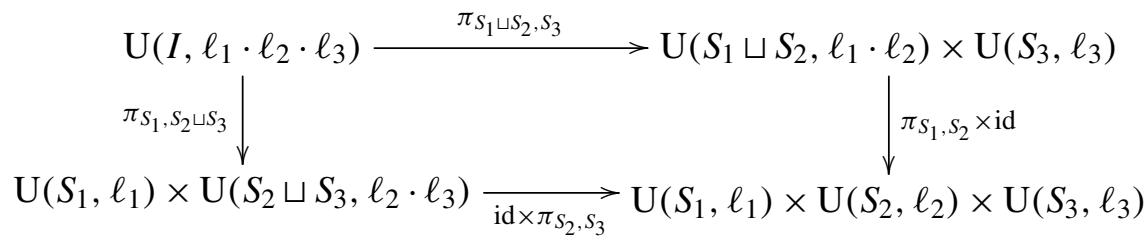

2.3. Direct sums and principal minors. The following key result relates the collection of morphisms $\sigma$ to the collection $\pi$ :

Lemma 3. Fix two decompositions $I=S_{1} \sqcup S_{2}=T_{1} \sqcup T_{2}$, and let $A, B, C$, and $D$ be the resulting intersections, as in (3). Let $\ell_{i}$ be a linear order on $S_{i}, i=1,2$, and $\ell=\ell_{1} \cdot \ell_{2}$. Then the following diagram commutes:

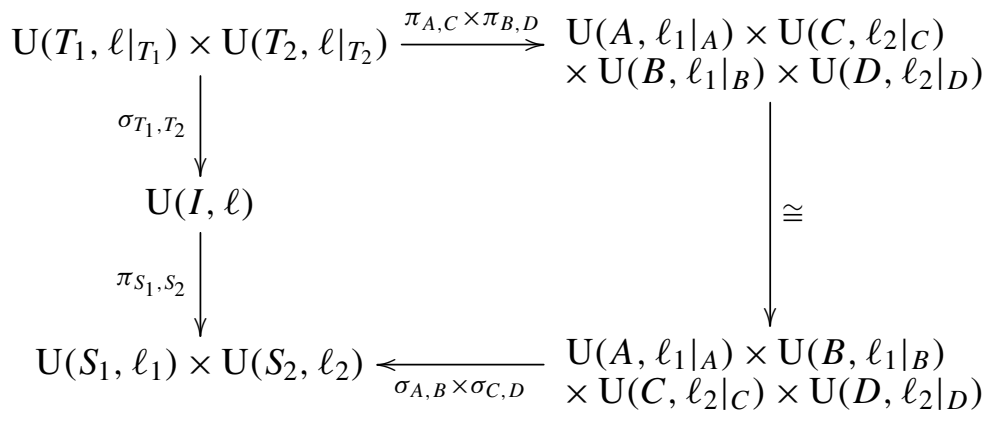

Proof. First note that since $\left.\ell\right|_{T_{1}}=\left(\left.\ell_{1}\right|_{A}\right) \cdot\left(\left.\ell_{2}\right|_{C}\right), \pi_{A, C}$ does map as stated in the diagram and similarly for $\pi_{B, D}$. The commutativity of the diagram boils down to the simple fact that

$$
(U \oplus V)_{S_{1}}=U_{A} \oplus V_{B}
$$

(and a similar statement for $S_{2}, C$, and $D$ ). This holds for any $U \in \mathrm{M}\left(T_{1}\right)$ and $V \in \mathrm{M}\left(T_{2}\right)$.

\section{A Hopf monoid of (class) functions}

We employ the operations of Section 2 (direct sum of matrices and the passage from a matrix to its principal minors) to build a Hopf monoid structure on the collection of function spaces on unitriangular matrices. The collection of class function spaces gives rise to a Hopf submonoid. With matrix entries in $\mathbb{F}_{2}$, the Hopf monoid of functions may be identified with the Hadamard product of the Hopf monoids of linear orders and of simple graphs. 
3.1. Functions. Given a set $X$, let $\mathbf{f}(X)$ denote the vector space of functions on $X$ with values on the base field $\mathbb{k}$. The functor

$$
f:\{\text { sets }\} \rightarrow\{\text { vector spaces }\}
$$

is contravariant. If at least one of two sets $X_{1}$ and $X_{2}$ is finite, then there is a canonical isomorphism

$$
\mathbf{f}\left(X_{1} \times X_{2}\right) \cong \mathbf{f}\left(X_{1}\right) \otimes \mathbf{f}\left(X_{2}\right) .
$$

A function $f \in \mathbf{f}\left(X_{1} \times X_{2}\right)$ corresponds to $\sum_{i} f_{i}^{1} \otimes f_{i}^{2} \in \mathbf{f}\left(X_{1}\right) \otimes \mathbf{f}\left(X_{2}\right)$ if and only if

$$
f\left(x_{1}, x_{2}\right)=\sum_{i} f_{i}^{1}\left(x_{1}\right) f_{i}^{2}\left(x_{2}\right) \quad \text { for all } x_{1} \in X_{1} \text { and } x_{2} \in X_{2} .
$$

Given an element $x \in X$, let $\kappa_{x}: X \rightarrow \mathbb{k}$ denote its characteristic function:

$$
\kappa_{x}(y)= \begin{cases}1 & \text { if } y=x \\ 0 & \text { if not. }\end{cases}
$$

Suppose now that $X$ is finite. As $x$ runs over the elements of $X$, the functions $\kappa_{x}$ form a linear basis of $f(X)$. If $\varphi: X \rightarrow X^{\prime}$ is a function and $x^{\prime}$ is an element of $X^{\prime}$, then

$$
\kappa_{x^{\prime}} \circ \varphi=\sum_{\varphi(x)=x^{\prime}} \kappa_{x} .
$$

Under (17),

$$
\kappa_{\left(x_{1}, x_{2}\right)} \leftrightarrow \kappa_{x_{1}} \otimes \kappa_{x_{2}} .
$$

3.2. Class functions on groups. Given a group $G$, let $\operatorname{cf}(G)$ denote the vector space of class functions on $G$. These are the functions $f: G \rightarrow \mathbb{k}$ that are constant on conjugacy classes of $G$. If $\varphi: G \rightarrow G^{\prime}$ is a morphism of groups and $f$ is a class function on $G^{\prime}$, then $f \circ \varphi$ is a class function on $G$. In this manner,

$$
\text { cf : }\{\text { groups }\} \rightarrow\{\text { vector spaces }\}
$$

is a contravariant functor. If at least one of two groups $G_{1}$ and $G_{2}$ is finite, then there is a canonical isomorphism

$$
\operatorname{cf}\left(G_{1} \times G_{2}\right) \cong \operatorname{cf}\left(G_{1}\right) \otimes \operatorname{cf}\left(G_{2}\right)
$$

obtained by restriction from the isomorphism (17).

Given a conjugacy class $C$ of $G$, let $\kappa_{C}: G \rightarrow \mathbb{k}$ denote its characteristic function:

$$
\kappa_{C}(x)= \begin{cases}1 & \text { if } x \in C, \\ 0 & \text { if not. }\end{cases}
$$


Suppose $G$ has finitely many conjugacy classes. As $C$ runs over the conjugacy classes of $G$, the functions $\kappa_{C}$ form a linear basis of $\operatorname{cf}(G)$. If $C^{\prime}$ is a conjugacy class of $G^{\prime}$, then

$$
\kappa_{C^{\prime}} \circ \varphi=\sum_{\varphi(C) \subseteq C^{\prime}} \kappa_{C} .
$$

The conjugacy classes of $G_{1} \times G_{2}$ are of the form $C_{1} \times C_{2}$, where $C_{i}$ is a conjugacy class of $G_{i}, i=1,2$. Under (21),

$$
\kappa_{C_{1} \times C_{2}} \leftrightarrow \kappa_{C_{1}} \otimes \kappa_{C_{2}} .
$$

3.3. Functions on unitriangular matrices. We assume for the rest of this section that the field $\mathbb{F}$ of matrix entries is finite. Thus, all groups $\mathrm{U}(I, \ell)$ of unitriangular matrices are finite.

We define a vector species $f(U)$ as follows. On a finite set $I$,

$$
\mathbf{f}(\mathrm{U})[I]=\bigoplus_{\ell \in \mathrm{L}[I]} \mathbf{f}(\mathrm{U}(I, \ell)) .
$$

In other words, $f(U)[I]$ is the direct sum of the spaces of functions on all unitriangular groups on $I$. A bijection $\sigma: I \cong J$ induces an isomorphism $\mathrm{U}(I, \ell) \cong \mathrm{U}(J, \sigma \cdot \ell)$ and therefore an isomorphism $\mathbf{f}(\mathrm{U})[I] \cong \mathbf{f}(\mathrm{U})[J]$. Thus, $\mathbf{f}(\mathrm{U})$ is a species.

Let $I=S_{1} \sqcup S_{2}$ and $\ell_{i} \in \mathrm{L}\left[S_{i}\right], i=1,2$. Applying the functor $\mathbf{f}$ to the morphism $\pi_{S_{1}, S_{2}}$ in (14) and composing with the isomorphism in (17), we obtain a linear map

$$
\mathbf{f}\left(\mathrm{U}\left(S_{1}, \ell_{1}\right)\right) \otimes \mathbf{f}\left(\mathrm{U}\left(S_{2}, \ell_{2}\right)\right) \rightarrow \mathbf{f}\left(\mathrm{U}\left(I, \ell_{1} \cdot \ell_{2}\right)\right) .
$$

Adding over all $\ell_{1} \in \mathrm{L}\left[S_{1}\right]$ and $\ell_{2} \in \mathrm{L}\left[S_{2}\right]$, we obtain a linear map

$$
\mu_{S_{1}, S_{2}}: \mathbf{f}(\mathrm{U})\left[S_{1}\right] \otimes \mathbf{f}(\mathrm{U})\left[S_{2}\right] \rightarrow \mathbf{f}(\mathrm{U})[I] .
$$

Explicitly, given functions $f: \mathrm{U}\left(S_{1}, \ell_{1}\right) \rightarrow \mathbb{k}$ and $g: \mathrm{U}\left(S_{2}, \ell_{2}\right) \rightarrow \mathbb{k}$,

$$
\mu_{S_{1}, S_{2}}(f \otimes g): \mathrm{U}\left(I, \ell_{1} \cdot \ell_{2}\right) \rightarrow \mathbb{k}
$$

is the function given by

$$
U \mapsto f\left(U_{S_{1}}\right) g\left(U_{S_{2}}\right)
$$

Similarly, from the map $\sigma_{S_{1}, S_{2}}$ in (11), we obtain the components

$$
\mathbf{f}(\mathrm{U}(I, \ell)) \rightarrow \mathbf{f}\left(\mathrm{U}\left(S_{1},\left.\ell\right|_{S_{1}}\right)\right) \otimes \mathbf{f}\left(\mathrm{U}\left(S_{2},\left.\ell\right|_{S_{2}}\right)\right)
$$

(one for each $\ell \in \mathrm{L}[I]$ ) of a linear map

$$
\Delta_{S_{1}, S_{2}}: \mathbf{f}(\mathrm{U})[I] \rightarrow \mathbf{f}(\mathrm{U})\left[S_{1}\right] \otimes \mathbf{f}(\mathrm{U})\left[S_{2}\right] .
$$


Explicitly, given a function $f: \mathrm{U}(I, \ell) \rightarrow \mathbb{k}$, we have $\Delta_{S_{1}, S_{2}}(f)=\sum_{i} f_{i}^{1} \otimes f_{i}^{2}$, where

$$
f_{i}^{1}: \mathrm{U}\left(S_{1},\left.\ell\right|_{S_{1}}\right) \rightarrow \mathbb{k} \quad \text { and } \quad f_{i}^{2}: \mathrm{U}\left(S_{2},\left.\ell\right|_{S_{2}}\right) \rightarrow \mathbb{k}
$$

are functions such that

$$
\begin{aligned}
& f\left(U_{1} \oplus U_{2}\right)=\sum_{i} f_{i}^{1}\left(U_{1}\right) f_{i}^{2}\left(U_{2}\right) \\
& \quad \text { for all } U_{1} \in \mathrm{U}\left(S_{1},\left.\ell\right|_{S_{1}}\right) \text { and } U_{2} \in \mathrm{U}\left(S_{2},\left.\ell\right|_{S_{2}}\right) .
\end{aligned}
$$

Proposition 4. With the operations (25) and (27), the species $\mathrm{f}(\mathrm{U})$ is a connected Hopf monoid. It is cocommutative.

Proof. Axioms (1), (2), and (4) follow from (12), (15), and (16) by functoriality. In the same manner, cocommutativity (5) follows from (13).

We describe the operations on the basis of characteristic functions (18). Let $U_{i} \in \mathrm{U}\left(S_{i}, \ell_{i}\right), i=1$, 2. It follows from (19) and (20), or from (26), that the product is

$$
\mu_{S_{1}, S_{2}}\left(\kappa_{U_{1}} \otimes \kappa_{U_{2}}\right)=\sum_{\pi_{S_{1}, S_{2}}(U)=\left(U_{1}, U_{2}\right)} \kappa_{U}=\sum_{\begin{array}{c}
U_{S_{1}}=U_{1} \\
U_{S_{2}}=U_{2}
\end{array}} \kappa_{U}
$$

Similarly, the coproduct is

$$
\Delta_{S_{1}, S_{2}}\left(\kappa_{U}\right)=\sum_{\sigma_{S_{1}, S_{2}}\left(U_{1}, U_{2}\right)=U} \kappa_{U_{1}} \otimes \kappa_{U_{2}}= \begin{cases}\kappa_{U_{S_{1}}} \otimes \kappa_{U_{S_{2}}} & \text { if } U=U_{S_{1}} \oplus U_{S_{2}}, \\ 0 & \text { otherwise. }\end{cases}
$$

3.4. Constant functions. Let $\mathbf{1}_{\ell}$ denote the constant function on $\mathrm{U}(I, \ell)$ with value 1 . Let $I=S_{1} \sqcup S_{2}$. It follows from (26) that

$$
\mu_{S_{1}, S_{2}}\left(\mathbf{1}_{\ell_{1}} \otimes \mathbf{1}_{\ell_{2}}\right)=\mathbf{1}_{\ell_{1} \cdot \ell_{2}}
$$

for any $\ell_{1} \in \mathrm{L}\left[S_{1}\right]$ and $\ell_{2} \in \mathrm{L}\left[S_{2}\right]$. Similarly, we see from (28) that

$$
\Delta_{S_{1}, S_{2}}\left(\mathbf{1}_{\ell}\right)=\mathbf{1}_{\ell_{\left.\right|_{1}}} \otimes \mathbf{1}_{\ell_{S_{2}}}
$$

for any $\ell \in \mathrm{L}[I]$. We thus have:

Corollary 5. The collection of maps

$$
L[I] \rightarrow \mathbf{f}(\mathrm{U})[I], \quad \ell \mapsto \mathbf{1}_{\ell}
$$

is an injective morphism of Hopf monoids. 
3.5. Class functions on unitriangular matrices. Let $\operatorname{cf}(\mathrm{U})[I]$ be the direct sum of the spaces of class functions on all unitriangular groups on $I$ :

$$
\operatorname{cf}(\mathrm{U})[I]=\bigoplus_{\ell \in \mathrm{L}[I]} \operatorname{cf}(\mathrm{U}(I, \ell))
$$

This defines a subspecies $\mathbf{c f}(\mathrm{U})$ of $\mathbf{f}(\mathrm{U})$.

Proceeding in the same manner as in Section 3.3, we obtain linear maps

$$
\operatorname{cf}(\mathrm{U})\left[S_{1}\right] \otimes \mathbf{c f}(\mathrm{U})\left[S_{2}\right] \frac{\mu_{S_{1}, S_{2}}}{\underset{\Delta_{S_{1}, S_{2}}}{\gtrless}} \operatorname{cf}(\mathrm{U})[I]
$$

by applying the functor $\mathrm{cf}$ to the morphisms $\pi_{S_{1}, S_{2}}$ and $\sigma_{S_{1}, S_{2}}$. This is meaningful since the latter are morphisms of groups (in the case of $\pi_{S_{1}, S_{2}}$, by Lemma 2).

Proposition 6. With these operations, the species $\mathrm{cf}(\mathrm{U})$ is a connected cocommutative Hopf monoid. It is a Hopf submonoid of $\mathbf{f}(\mathrm{U})$.

Proof. As in the proof of Proposition 4, the first statement follows by functoriality. The second follows from the naturality of the inclusion of class functions and its compatibility with the isomorphisms in (17) and (21).

We describe the operations on the basis of characteristic functions (22). Let $C_{i}$ be a conjugacy class of $\mathrm{U}\left(S_{i}, \ell_{i}\right), i=1,2$. It follows from (23) and (24) that the product is

$$
\mu_{S_{1}, S_{2}}\left(\kappa_{C_{1}} \otimes \kappa_{C_{2}}\right)=\sum_{\pi_{S_{1}, S_{2}}(C) \subseteq C_{1} \times C_{2}} \kappa_{C},
$$

where the sum is over conjugacy classes $C$ in $\mathrm{U}\left(I, \ell_{1} \cdot \ell_{2}\right)$. Similarly, the coproduct is

$$
\Delta_{S_{1}, S_{2}}\left(\kappa_{C}\right)=\sum_{\sigma_{S_{1}, S_{2}}\left(C_{1} \times C_{2}\right) \subseteq C} \kappa_{C_{1}} \otimes \kappa_{C_{2}}
$$

Here $C$ is a conjugacy class of $\mathrm{U}(I, \ell)$, and the sum is over pairs of conjugacy classes $C_{i}$ of $\mathrm{U}\left(S_{i},\left.\ell\right|_{S_{i}}\right)$.

\section{Remark 7. Let}

$$
\mathscr{F}:\{\text { groups }\} \rightarrow\{\text { vector spaces }\}
$$

be a functor that is contravariant and bilax monoidal in the sense of [Aguiar and Mahajan 2010, Section 3.1]. The construction of the Hopf monoids $f(U)$ and $\operatorname{cf}(\mathrm{U})$ can be carried out for any such functor $\mathscr{F}$ in place of cf in exactly the same manner. It can also be carried out for a covariant bilax monoidal functor $\mathscr{F}$ in a similar manner. 
3.6. A combinatorial model. To a unitriangular matrix $U \in \mathrm{U}(I, \ell)$, we associate a graph $g(U)$ on $I$ as follows: there is an edge between $i$ and $j$ if $i<j$ in $\ell$ and $u_{i j} \neq 0$. For example, given nonzero entries $a, b, c \in \mathbb{F}$,

$$
\ell=h i j k, \quad U=\left(\begin{array}{rrrr}
1 & 0 & 0 & a \\
& 1 & b & c \\
& & 1 & 0 \\
& & & 1
\end{array}\right) \Longrightarrow g(U)=\bullet_{h}
$$

Recall the Hopf monoids $\boldsymbol{L}$ and $\boldsymbol{G}$ and the notion of Hadamard product from Section 1. Let

$$
\phi: L \times G \rightarrow f(U)
$$

be the map with components

$$
(L \times G)[I] \rightarrow \mathbf{f}(\mathrm{U})[I]
$$

given as follows. On a basis element $\ell \otimes m_{g} \in \boldsymbol{L}[I] \otimes \boldsymbol{G}[I]=(\boldsymbol{L} \times \boldsymbol{G})[I]$, we set

$$
\phi\left(\ell \otimes m_{g}\right)=\sum_{\substack{U \in \mathrm{U}(I, \ell) \\ g(U)=g}} \kappa_{U} \in \mathbf{f}(\mathrm{U}(I, \ell)) \subseteq \mathbf{f}(\mathrm{U})[I]
$$

and extend by linearity. The map relates the $m$-basis of $\boldsymbol{G}$ to the basis of characteristic functions (18) of $\mathbf{f}(\mathrm{U})$.

Proposition 8. Let $\mathbb{F}$ be an arbitrary finite field. The map $\phi: \boldsymbol{L} \times \boldsymbol{G} \rightarrow \mathbf{f}(\mathrm{U})$ is an injective morphism of Hopf monoids.

Proof. From the definition of the Hopf monoid operations on a Hadamard product and formulas (6) and (9), it follows that

$$
\mu_{S_{1}, S_{2}}\left(\left(\ell_{1} \otimes m_{g_{1}}\right) \otimes\left(\ell_{2} \otimes m_{g_{2}}\right)\right)=\sum_{\substack{g\left|S_{1}=g_{1} \\ g\right| S_{2}=g_{2}}} \ell_{1} \cdot \ell_{2} \otimes m_{g} .
$$

Comparing with (29), we see that products are preserved since given $U \in \mathrm{U}(I, \ell)$, we have

$$
g\left(U_{S_{i}}\right)=\left.g(U)\right|_{S_{i}}
$$

The verification for coproducts is similar, employing (6), (10), and (30) and the fact that given $I=S_{1} \sqcup S_{2}$ and $U_{i} \in \mathrm{U}\left(S_{i},\left.\ell\right|_{S_{i}}\right)$, we have

$$
g\left(U_{1} \oplus U_{2}\right)=g\left(U_{1}\right) \sqcup g\left(U_{2}\right) .
$$

Consider the map $\psi: \mathbf{f}(\mathrm{U}) \rightarrow \boldsymbol{L} \times \boldsymbol{G}$ given by

$$
\psi\left(\kappa_{U}\right)=\ell \otimes m_{g(U)}
$$


for any $U \in \mathrm{U}(I, \ell)$. Then

$$
\psi \phi\left(\ell \otimes m_{g}\right)=(q-1)^{e(g)} \ell \otimes m_{g},
$$

where $q$ is the cardinality of $\mathbb{F}$ and $e(g)$ is the number of edges in $g$. Thus, $\phi$ is injective.

We mention that the map $\psi$ in (35) is a morphism of comonoids but not of monoids in general.

Assume now that the matrix entries are from $\mathbb{F}_{2}$, the field with two elements. In this case, the matrix $U$ is uniquely determined by the linear order $\ell$ and the graph $g(U)$. Therefore, the map $\phi$ is invertible with inverse $\psi$.

Corollary 9. There is an isomorphism of Hopf monoids

$$
\mathrm{f}(\mathrm{U}) \cong \boldsymbol{L} \times \boldsymbol{G}
$$

between the Hopf monoid of functions on unitriangular matrices with entries in $\mathbb{F}_{2}$ and the Hadamard product of the Hopf monoids of linear orders and simple graphs.

On an arbitrary function $f: \mathrm{U}(I, \ell) \rightarrow \mathbb{k}$, the isomorphism is given by

$$
\psi(f)=\ell \otimes \sum_{U \in \mathrm{U}(I, \ell)} f(U) m_{g(U)} .
$$

The coefficients of the $m$-basis elements are the values of $f$.

\section{A Hopf monoid of superclass functions}

An abstract notion of superclass (and supercharacter) has been introduced by Diaconis and Isaacs [2008]. We only need a minimal amount of related concepts that we review in Sections 4.1 and 4.2. For this purpose, we first place ourselves in the setting of algebra groups. In Section 4.2, we construct a Hopf monoid structure on the collection of spaces of superclass functions on the unitriangular groups by the same procedure as that in Section 3. The combinatorics of these superclasses is understood from the thesis of Yan [2001] (reviewed in slightly different terms in Section 4.3), and this allows us to obtain an explicit description for the Hopf monoid operations in Section 4.4. This leads to a theorem in Section 4.5 identifying the Hopf monoid of superclass functions with matrix entries in $\mathbb{F}_{2}$ to the Hadamard product of the Hopf monoids of linear orders and set partitions. The combinatorial models for functions and for superclass functions are related in Section 4.6.

4.1. Superclass functions on algebra groups. Let $\mathfrak{n}$ be a nilpotent algebra: an associative, nonunital algebra in which every element is nilpotent. Let $\overline{\mathfrak{n}}=\mathbb{F} \oplus \mathfrak{n}$ denote the result of adjoining a unit to $\mathfrak{n}$. The set

$$
G(\mathfrak{n})=\{1+n \mid n \in \mathfrak{n}\}
$$


is a subgroup of the group of invertible elements of $\overline{\mathfrak{n}}$. A group of this form is called an algebra group. (This is the terminology employed in [Diaconis and Isaacs 2008] and, in a slightly different context, [André 1999; Isaacs 1995].)

A morphism of nilpotent algebras $\varphi: \mathfrak{m} \rightarrow \mathfrak{n}$ has a unique unital extension $\overline{\mathfrak{m}} \rightarrow \overline{\mathfrak{n}}$, and this sends $G(\mathfrak{m})$ to $G(\mathfrak{n})$. A morphism of algebra groups is a map of this form.

Warning. When we refer to the algebra group $G(\mathfrak{n})$, it is implicitly assumed that the algebra $\mathfrak{n}$ is given as well.

Following Yan [2001], we define an equivalence relation on $G(\mathfrak{n})$ as follows. Given $x, y \in G(\mathfrak{n})$, we write $x \sim y$ if there exist $g, h \in G(\mathfrak{n})$ such that

$$
y-1=g(x-1) h .
$$

Following now Diaconis and Isaacs [2008], we refer to the equivalence classes of this relation as superclasses and to the functions $G(\mathfrak{n}) \rightarrow \mathbb{k}$ constant on these classes as superclass functions. The set of such functions is denoted $\operatorname{scf}(G(\mathfrak{n}))$.

Since

$$
g x g^{-1}-1=g(x-1) g^{-1},
$$

we have that $x \sim g x g^{-1}$ for any $x, g \in G(\mathfrak{n})$. Thus, each superclass is a union of conjugacy classes, and hence, every superclass function is a class function:

$$
\operatorname{scf}(G(\mathfrak{n})) \subseteq \operatorname{cf}(G(\mathfrak{n}))
$$

A morphism $\varphi: G(\mathfrak{m}) \rightarrow G(\mathfrak{n})$ of algebra groups preserves the relation $\sim$. Therefore, if $f: G(\mathfrak{n}) \rightarrow \mathbb{k}$ is a superclass function on $G(\mathfrak{n})$, then $f \circ \varphi$ is a superclass function on $G(\mathfrak{m})$. In this manner,

$$
\text { scf : }\{\text { algebra groups }\} \rightarrow\{\text { vector spaces }\}
$$

is a contravariant functor. In addition, the inclusion (37) is natural with respect to morphisms of algebra groups.

The direct product of two algebra groups is another algebra group. Indeed,

$$
G\left(\mathfrak{n}_{1}\right) \times G\left(\mathfrak{n}_{2}\right) \cong G\left(\mathfrak{n}_{1} \oplus \mathfrak{n}_{2}\right)
$$

and $\mathfrak{n}_{1} \oplus \mathfrak{n}_{2}$ is nilpotent. Moreover,

$$
\left(x_{1}, x_{2}\right) \sim\left(y_{1}, y_{2}\right) \Longleftrightarrow\left(x_{1} \sim y_{1} \text { and } x_{2} \sim y_{2}\right) .
$$

Therefore, a superclass of the product is a pair of superclasses from the factors, and if at least one of the two groups is finite, there is a canonical isomorphism

$$
\operatorname{scf}\left(G\left(\mathfrak{n}_{1}\right) \times G\left(\mathfrak{n}_{2}\right)\right) \cong \operatorname{scf}\left(G\left(\mathfrak{n}_{1}\right)\right) \otimes \operatorname{scf}\left(G\left(\mathfrak{n}_{2}\right)\right) .
$$


4.2. Superclass functions on unitriangular matrices. Given a finite set $I$ and a linear order $\ell$ on $I$, let $\mathfrak{n}(I, \ell)$ denote the subalgebra of $\mathrm{M}(I)$ consisting of strictly upper-triangular matrices

$$
N=\left(n_{i j}\right)_{i, j \in I}, \quad n_{i j}=0 \text { whenever } i \geq_{\ell} j .
$$

Then $\mathfrak{n}(I, \ell)$ is nilpotent and $G(\mathfrak{n}(I, \ell))=\mathrm{U}(I, \ell)$. Thus, the unitriangular groups are algebra groups.

We assume from now on that the field $\mathbb{F}$ is finite.

We define, for each finite set $I$,

$$
\operatorname{scf}(\mathrm{U})[I]=\bigoplus_{\ell \in \mathrm{L}[I]} \operatorname{scf}(\mathrm{U}(I, \ell)) .
$$

This defines a species $\operatorname{scf}(\mathrm{U})$. Proceeding in the same manner as in Sections 3.3 and 3.5, we obtain linear maps

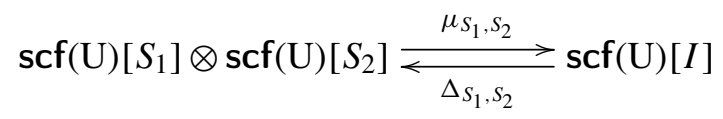

by applying the functor scf to the morphisms $\pi_{S_{1}, S_{2}}$ and $\sigma_{S_{1}, S_{2}}$. This is meaningful since the latter are morphisms of algebra groups: it was noted in Section 2.1 that $\sigma_{S_{1}, S_{2}}$ is the restriction of a morphism defined on the full matrix algebras while the considerations of Lemma 2 show that $\pi_{S_{1}, S_{2}}$ is the restriction of a morphism defined on the algebra of upper-triangular matrices.

Proposition 10. With these operations, the species $\operatorname{scf}(\mathrm{U})$ is a connected cocommutative Hopf monoid. It is a Hopf submonoid of $\mathrm{cf}(\mathrm{U})$.

Proof. As in the proof of Proposition 4, the first statement follows by functoriality. The second follows from the naturality of the inclusion (37).

(31) and (32) continue to hold for the (co)product of superclass functions.

The constant function $\mathbf{1}_{\ell}$ is a superclass function. Thus, the morphism of Hopf monoids of Corollary 5 factors through $\operatorname{scf}(\mathrm{U})$ and $\operatorname{cf}(\mathrm{U})$ :

$$
L \hookrightarrow \operatorname{scf}(U) \hookrightarrow \operatorname{cf}(U) \hookrightarrow f(U) .
$$

4.3. Combinatorics of the superclasses. Yan [2001] showed superclasses are parametrized by certain combinatorial data essentially along the lines presented below.

According to (36), two unitriangular matrices $U_{1}$ and $U_{2}$ are in the same superclass if and only if $U_{2}-\mathrm{Id}$ is obtained from $U_{1}-\mathrm{Id}$ by a sequence of elementary row and column operations. The available operations are from the unitriangular group itself, so the pivot entries cannot be normalized. Thus, each superclass contains a unique matrix $U$ such that $U-$ Id has at most one nonzero entry in each row 
and each column. We refer to this matrix $U$ as the canonical representative of the superclass.

We proceed to encode such representatives in terms of combinatorial data.

We first discuss the combinatorial data. Let $\ell$ be a linear order on a finite set $I$ and $X$ a partition of $I$. Let us say that $i, j \in I$ bound an arc if

- $i$ precedes $j$ in $\ell$,

- $i$ and $j$ are in the same block of $X$, say $S$, and

- no other element of $S$ lies between $i$ and $j$ in the order $\ell$.

The set of $\operatorname{arcs}$ is

$$
A(X, \ell):=\{(i, j) \mid i \text { and } j \text { bound an } \operatorname{arc}\} .
$$

Consider also a function

$$
\alpha: A(X, \ell) \rightarrow \mathbb{F}^{\times}
$$

from the set of arcs to the nonzero elements of $\mathbb{F}$. We say that the pair $(X, \alpha)$ is an arc diagram on the linearly ordered set $(I, \ell)$. We may visualize an arc diagram:

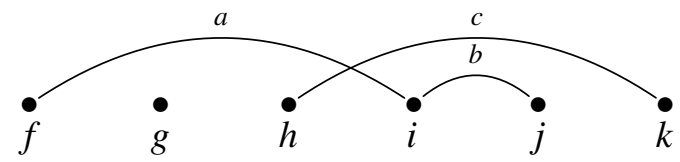

Here the combinatorial data is

$\ell=f g h i j k, \quad X=\{\{f, i, j\},\{g\},\{h, k\}\}, \quad \alpha(f, i)=a, \alpha(i, j)=b, \alpha(h, k)=c$.

Fix the linear order $\ell$. To an arc diagram $(X, \alpha)$ on $(I, \ell)$, we associate a matrix $U_{X, \alpha}$ with entries

$$
u_{i j}= \begin{cases}\alpha(i, j) & \text { if }(i, j) \in A(X, \ell) \\ 1 & \text { if } i=j \\ 0 & \text { otherwise. }\end{cases}
$$

Clearly, the matrix $U_{X, \alpha}$ is $\ell$-unitriangular and $U_{X, \alpha}-\mathrm{Id}$ has at most one nonzero entry in each row and each column. In the above example,

$$
U_{X, \alpha}=\left(\begin{array}{ccccccc}
1 & 0 & 0 & a & 0 & 0 \\
& 1 & 0 & 0 & 0 & 0 \\
& & 1 & 0 & 0 & c \\
& & & 1 & d & 0 \\
& & & & & 1 & 0 \\
& & & & & & 1
\end{array}\right) .
$$

Conversely, any canonical representative matrix $U \in \mathrm{U}(I, \ell)$ is of the form $U_{X, \alpha}$ for a unique arc diagram $(X, \alpha)$ on $(I, \ell)$ : the location of the nonzero entries 
determines the set of arcs, and the values of the entries determine the function $\alpha$. The smallest equivalence relation on $I$ containing the set of arcs determines the partition $X$.

In conclusion, the canonical representatives, and hence the superclasses, are in bijection with the set of arc diagrams. We let $C_{X, \alpha}$ denote the superclass of $\mathrm{U}(I, \ell)$ containing $U_{X, \alpha}$, and we write $\kappa_{X, \alpha}$ for the characteristic function of this class. As $(X, \alpha)$ runs over all arc diagrams on $(I, \ell)$, these functions form a basis of the space $\operatorname{scf}(\mathrm{U}(I, \ell))$.

We describe principal minors and direct sums of the canonical representatives. To this end, fix $\ell \in \mathrm{L}[I]$ and recall the notions of union and restriction of set partitions discussed in Section 1.3.

Let $S \subseteq I$ be an arbitrary subset. Given a partition $X$ of $I$, let $\left.A(X, \ell)\right|_{S}$ denote the subset of $A(X, \ell)$ consisting of those $\operatorname{arcs}(i, j)$ where both $i$ and $j$ belong to $S$. We let $\left.\alpha\right|_{S}$ denote the restriction of $\alpha$ to $\left.A(X, \ell)\right|_{S}$. We have $\left.A(X, \ell)\right|_{S} \subseteq A\left(\left.X\right|_{S},\left.\ell\right|_{S}\right)$, and if $S$ is an $\ell$-segment, then

$$
\left.A(X, \ell)\right|_{S}=A\left(\left.X\right|_{S},\left.\ell\right|_{S}\right)
$$

In this case, we obtain an arc diagram $\left(\left.X\right|_{S},\left.\alpha\right|_{S}\right)$ on $\left(S,\left.\ell\right|_{S}\right)$, and we have

$$
\left(U_{X, \alpha}\right)_{S}=U_{\left.X\right|_{S},\left.\alpha\right|_{S}} .
$$

Suppose now that $I=S_{1} \sqcup S_{2}$ and $\left(X_{i}, \alpha_{i}\right)$ is an arc diagram on $\left(S_{i},\left.\ell\right|_{S_{i}}\right), i=1,2$. Then

$$
A\left(X_{1} \sqcup X_{2}, \ell\right)=A\left(X_{1},\left.\ell\right|_{S_{1}}\right) \sqcup A\left(X_{2},\left.\ell\right|_{S_{2}}\right) .
$$

Let $\alpha_{1} \sqcup \alpha_{2}$ denote the common extension of $\alpha_{1}$ and $\alpha_{2}$ to this set. Then the pair $\left(X_{1} \sqcup X_{2}, \alpha_{1} \sqcup \alpha_{2}\right)$ is then an arc diagram on $(I, \ell)$ and

$$
U_{X_{1}, \alpha_{1}} \oplus U_{X_{2}, \alpha_{2}}=U_{X_{1} \sqcup X_{2}, \alpha_{1} \sqcup \alpha_{2}} .
$$

4.4. Combinatorics of the (co)product. We now describe the product and coproduct of the Hopf monoid $\operatorname{scf}(\mathrm{U})$ on the basis $\left\{\kappa_{X, \alpha}\right\}$ of Section 4.3. We employ (31) and (32), which, as discussed in Section 4.2, hold for superclass functions.

Let $I=S_{1} \sqcup S_{2}$ and $\ell_{i} \in \mathrm{L}\left[S_{i}\right], i=1,2$, and consider the product

$$
\operatorname{scf}\left(\mathrm{U}\left(S_{1}, \ell_{1}\right)\right) \times \operatorname{scf}\left(\mathrm{U}\left(S_{2}, \ell_{2}\right)\right) \rightarrow \operatorname{scf}\left(\mathrm{U}\left(I, \ell_{1} \cdot \ell_{2}\right)\right) .
$$

Let $\left(X_{i}, \alpha_{i}\right)$ be an arc diagram on $\left(I, \ell_{i}\right), i=1,2$. According to (31), we have

$$
\mu_{S_{1}, S_{2}}\left(\kappa_{X_{1}, \alpha_{1}} \otimes \kappa_{X_{2}, \alpha_{2}}\right)=\sum_{\pi_{S_{1}, S_{2}}\left(C_{X, \alpha}\right) \subseteq C_{X_{1}, \alpha_{1}} \times C_{X_{2}, \alpha_{2}}} \kappa_{X, \alpha},
$$


a sum over arc diagrams $(X, \alpha)$ on $\left(I, \ell_{1} \cdot \ell_{2}\right)$. Since $\pi_{S, T}$ preserves superclasses,

$$
\begin{aligned}
\pi_{S_{1}, S_{2}}\left(C_{X, \alpha}\right) \subseteq C_{X_{1}, \alpha_{1}} \times C_{X_{2}, \alpha_{2}} & \Longleftrightarrow \pi_{S_{1}, S_{2}}\left(U_{X, \alpha}\right) \in C_{X_{1}, \alpha_{1}} \times C_{X_{2}, \alpha_{2}} \\
& \Longleftrightarrow\left(U_{X, \alpha}\right)_{S_{i}} \in C_{X_{i}, \alpha_{i}}, \quad i=1,2 .
\end{aligned}
$$

In view of (39), this is in turn equivalent to

$$
\left.X\right|_{S_{i}}=X_{i} \quad \text { and }\left.\quad \alpha\right|_{S_{i}},=\alpha_{i}, \quad i=1,2 .
$$

In conclusion,

$$
\mu_{S_{1}, S_{2}}\left(\kappa_{X_{1}, \alpha_{1}} \otimes \kappa_{X_{2}, \alpha_{2}}\right)=\sum_{\substack{\left.X\right|_{S_{i}}=\left.X_{i} \\ \alpha\right|_{S_{i}}=\alpha_{i}}} \kappa_{X, \alpha}
$$

The sum is over all arc diagrams $(X, \alpha)$ on $\left(I, \ell_{1} \cdot \ell_{2}\right)$ whose restriction to $S_{i}$ is $\left(X_{i}, \alpha_{i}\right)$ for $i=1,2$.

Take now $\ell \in \mathrm{L}[I], I=S_{1} \sqcup S_{2}$, and consider the coproduct

$$
\operatorname{scf}(\mathrm{U}(I, \ell)) \rightarrow \operatorname{scf}\left(\mathrm{U}\left(S_{1},\left.\ell\right|_{S_{1}}\right)\right) \times \operatorname{scf}\left(\mathrm{U}\left(S_{2},\left.\ell\right|_{S_{2}}\right)\right) .
$$

Let $(X, \alpha)$ be an arc diagram on $(I, \ell)$. According to (32), we have

$$
\Delta_{S_{1}, S_{2}}\left(\kappa_{X, \alpha}\right)=\sum_{\sigma_{S_{1}, S_{2}}\left(C_{X_{1}, \alpha_{1}} \times C_{X_{2}, \alpha_{2}}\right) \subseteq C_{X, \alpha}} \kappa_{X_{1}, \alpha_{1}} \otimes \kappa_{X_{2}, \alpha_{2}},
$$

a sum over arc diagrams $\left(X_{i}, \alpha_{i}\right)$ on $\left(S_{i},\left.\ell\right|_{S_{i}}\right)$. The superclass $C_{X_{1}, \alpha_{1}} \times C_{X_{2}, \alpha_{2}}$ contains $\left(U_{X_{1}, \alpha_{1}}, U_{X_{2}, \alpha_{2}}\right)$, and hence, its image under $\sigma_{S_{1}, S_{2}}$ contains

$$
U_{X_{1}, \alpha_{1}} \oplus U_{X_{2}, \alpha_{2}}=U_{X_{1} \sqcup X_{2}, \alpha_{1} \sqcup \alpha_{2}}
$$

by (41). Therefore,

$$
\sigma_{S_{1}, S_{2}}\left(C_{X_{1}, \alpha_{1}} \times C_{X_{2}, \alpha_{2}}\right) \subseteq C_{X, \alpha} \Longleftrightarrow X_{1} \sqcup X_{2}=X \quad \text { and } \quad \alpha_{1} \sqcup \alpha_{2}=\alpha .
$$

Note that $X_{1} \sqcup X_{2}=X$ if and only if $S_{1}$ (or equivalently, $S_{2}$ ) is a union of blocks of $X$. In this case, $X_{i}=\left.X\right|_{S_{i}}$ and $\alpha_{i}=\left.\alpha\right|_{S_{i}}$. In conclusion,

$$
\Delta_{S_{1}, S_{2}}\left(\kappa_{X, \alpha}\right)= \begin{cases}\kappa_{X\left|S_{1}, \alpha\right|_{S_{1}}} \otimes \kappa_{X\left|S_{2}, \alpha\right|_{S_{2}}} & \text { if } S_{1} \text { is the union of some blocks of } X, \\ 0 & \text { otherwise. }\end{cases}
$$

4.5. Decomposition as a Hadamard product. The apparent similarity between the combinatorial description of the Hopf monoid operations of $\operatorname{scf}(\mathrm{U})$ in Section 4.4 and those of the Hopf monoids $\boldsymbol{L}$ and $\boldsymbol{\Pi}$ in Sections 1.2 and 1.3 can be formalized. Recall the Hadamard product of Hopf monoids from Section 1.5.

Let

$$
\phi: L \times \Pi \rightarrow \operatorname{scf}(\mathrm{U})
$$


be the map with components

$$
(L \times \Pi)[I] \rightarrow \operatorname{scf}(\mathrm{U})[I]
$$

given as follows. On a basis element $\ell \otimes m_{X} \in \boldsymbol{L}[I] \otimes \boldsymbol{\Pi}[I]=(\boldsymbol{L} \times \boldsymbol{\Pi})[I]$, we set

$$
\phi\left(\ell \otimes m_{X}\right)=\sum_{\alpha: A(X, \ell) \rightarrow \mathbb{F}^{\times}} \kappa_{X, \alpha} \in \operatorname{scf}(\mathrm{U}(I, \ell)) \subseteq \operatorname{scf}(\mathrm{U})[I]
$$

and extend by linearity. The morphism $\phi$ adds labels to the arcs in all possible ways.

Proposition 11. Let $\mathbb{F}$ be an arbitrary finite field. The map $\phi: L \times \Pi \rightarrow \operatorname{scf}(\mathrm{U})$ is an injective morphism of Hopf monoids.

Proof. This follows by comparing definitions, as in the proof of Proposition 8 . The relevant equations are (6), (7), and (8) for the operations of $\boldsymbol{L} \times \boldsymbol{\Pi}$ and (42) and (43) for the operations of $\operatorname{scf}(\mathrm{U})$.

When the field of matrix entries is $\mathbb{F}_{2}$, the arc labels are uniquely determined. The map $\phi$ is then invertible with inverse $\psi$ given by

$$
\psi\left(\kappa_{X, \alpha}\right)=\ell \otimes m_{X}
$$

for any arc diagram $(X, \alpha)$ on a linearly ordered set $(I, \ell)$. We thus have:

Corollary 12. There is an isomorphism of Hopf monoids

$$
\operatorname{scf}(\mathrm{U}) \cong L \times \Pi
$$

between the Hopf monoid of superclass functions on unitriangular matrices with entries in $\mathbb{F}_{2}$ and the Hadamard product of the Hopf monoids of linear orders and set partitions.

4.6. Relating the combinatorial models. The results of Section 4.5 provide a combinatorial model for the Hopf monoid $\operatorname{scf}(\mathrm{U})$. They parallel those of Section 3.6 that do the same for $f(U)$. We now interpret the inclusion $\operatorname{scf}(U) \hookrightarrow f(U)$ in these terms.

Let $X$ be a partition on a linearly ordered set $(I, \ell)$. We may regard the set of $\operatorname{arcs} A(X, \ell)$ as a simple graph on $I$. Let $G(X, \ell)$ denote the set of simple graphs $g$ on $I$ such that

- $g$ contains the graph $A(X, \ell)$ and

- if $i<j$ in $\ell$ and $g \backslash A(X, \ell)$ contains an edge between $i$ and $j$, then there exists $k$ such that

$$
i<k<j \text { in } \ell \text { and either }(i, k) \in A(X, \ell) \text { or }(k, j) \in A(X, \ell) \text {. }
$$


The following illustrates the extra edges (dotted) that may be present in $g$ when an arc (solid) is present in $A(X, \ell)$ :

Define a map

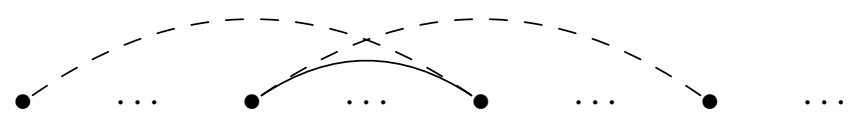

$$
L \times \Pi \rightarrow L \times G
$$

with components

$$
(\boldsymbol{L} \times \boldsymbol{\Pi})[I] \rightarrow(\boldsymbol{L} \times \boldsymbol{G})[I], \quad \ell \otimes m_{X} \mapsto \ell \otimes \sum_{g \in G(X, \ell)} m_{g} .
$$

Proposition 13. The map $\boldsymbol{L} \times \boldsymbol{\Pi} \rightarrow \boldsymbol{L} \times \boldsymbol{G}$ is an injective morphism of Hopf monoids. Moreover, the following diagram commutes:

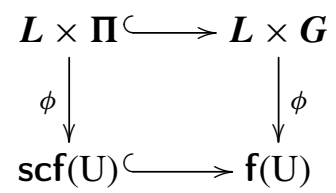

Proof. It is enough to prove the commutativity of the diagram since all other maps in the diagram are injective morphisms. The commutativity boils down to the following fact. Given $X \in \Pi[I], \ell \in \mathrm{L}[I]$, and $U \in \mathrm{U}(I, \ell)$,

$$
U \in C_{X, \alpha} \text { for some } \alpha: A(X, \ell) \rightarrow \mathbb{F}^{\times} \Longleftrightarrow g(U) \in G(X, \ell) \text {. }
$$

This expresses the fact that a matrix $U$ belongs to the superclass $C_{X, \alpha}$ if and only if the nonzero entries of $U-\mathrm{Id}$ are located either above or to the right of the nonzero entries of the representative $U_{X, \alpha}$.

\section{Freeness}

We prove that the Hopf monoids $f(U)$ and $\operatorname{scf}(U)$ are free and the Hopf structure is isomorphic to the canonical one on a free monoid. We assume that the base field $\mathbb{k}$ is of characteristic 0, which enables us to apply the results of the Appendix.

5.1. A partial order on arc diagrams. Let $(I, \ell)$ be a linearly ordered set. Given arc diagrams $(X, \alpha)$ and $(Y, \beta)$ on $(I, \ell)$, we write

$$
(X, \alpha) \leq(Y, \beta)
$$

if

$$
A(X, \ell) \subseteq A(Y, \ell) \quad \text { and }\left.\quad \beta\right|_{A(X, \ell)}=\alpha .
$$


In other words, every arc of $X$ is an arc of $Y$ and with the same label. In particular, the partition $Y$ is coarser than $X$. On the other hand, the following arc diagrams are incomparable (regardless of the labels) even though the partition on the right is the coarsest one:
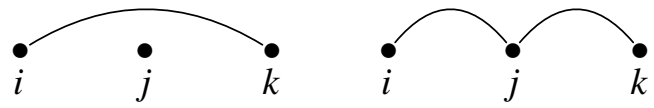

The poset of arc diagrams has a unique minimum (the partition into singletons, for which there are no arcs) but several maximal elements. The arc diagrams above are the two maximal elements when $\ell=i j k$ (up to a choice of labels).

A partition $X$ of the linearly ordered set $(I, \ell)$ is atomic if no proper initial $\ell$-segment of $I$ is a union of blocks of $X$. Equivalently, there is no decomposition $I=S_{1} \sqcup S_{2}$ into proper $\ell$-segments such that $X=\left.\left.X\right|_{S_{1}} \sqcup X\right|_{S_{2}}$.

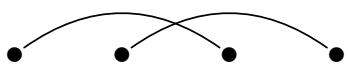

atomic

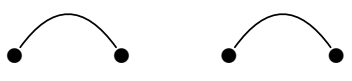

nonatomic

If $(X, \alpha)$ is a maximal element of the poset of arc diagrams, then $X$ is an atomic partition. But if $X$ is atomic, $(X, \alpha)$ need not be maximal (regardless of $\alpha$ ).
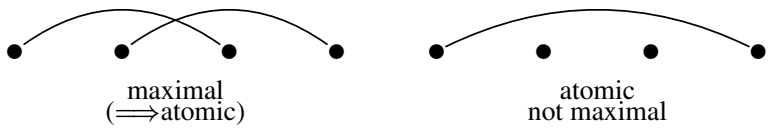

5.2. A second basis for $\operatorname{scf}(\mathbf{U})$. We employ the partial order of Section 5.1 to define a new basis $\left\{\lambda_{X, \alpha}\right\}$ of $\operatorname{scf}(\mathrm{U}(I, \ell))$ by

$$
\lambda_{X, \alpha}=\sum_{(X, \alpha) \leq(Y, \beta)} \kappa_{Y, \beta} .
$$

The product of the Hopf monoid $\operatorname{scf}(\mathrm{U})$ takes a simple form on the $\lambda$-basis.

Proposition 14. Let $I=S_{1} \sqcup S_{2}$ and $\ell_{i} \in \mathrm{L}\left[S_{i}\right], i=1,2$. Then

$$
\mu_{S_{1}, S_{2}}\left(\lambda_{X_{1}, \alpha_{1}} \otimes \lambda_{X_{2}, \alpha_{2}}\right)=\lambda_{X_{1} \sqcup X_{2}, \alpha_{1} \sqcup \alpha_{2}}
$$

for any arc diagrams $\left(X_{i}, \alpha_{i}\right)$ on $\left(S_{i}, \ell_{i}\right), i=1,2$.

Proof. We calculate using (42) and (45):

$$
\mu_{S_{1}, S_{2}}\left(\lambda_{X_{1}, \alpha_{1}} \otimes \lambda_{X_{2}, \alpha_{2}}\right)=\sum_{\left(X_{i}, \alpha_{i}\right) \leq\left(Y_{i}, \beta_{i}\right)} \mu_{S_{1}, S_{2}}\left(\kappa_{Y_{1}, \beta_{1}} \otimes \kappa_{Y_{2}, \beta_{2}}\right)=\sum_{\left(X_{i}, \alpha_{i}\right) \leq\left(\left.Y\right|_{S_{i}},\left.\beta\right|_{S_{i}}\right)} \kappa_{Y, \beta} .
$$

Now by (38) and (40), we have

$$
\left(X_{i}, \alpha_{i}\right) \leq\left(\left.Y\right|_{S_{i}},\left.\beta\right|_{S_{i}}\right), i=1,2 \Longleftrightarrow\left(X_{1} \sqcup X_{2}, \alpha_{1} \sqcup \alpha_{2}\right) \leq(Y, \beta) .
$$


Therefore,

$$
\mu_{S_{1}, S_{2}}\left(\lambda_{X_{1}, \alpha_{1}} \otimes \lambda_{X_{2}, \alpha_{2}}\right)=\sum_{\left(X_{1} \sqcup X_{2}, \alpha_{1} \sqcup \alpha_{2}\right) \leq(Y, \beta)} \kappa_{Y, \beta}=\lambda_{X_{1} \sqcup X_{2}, \alpha_{1} \sqcup \alpha_{2}} .
$$

The coproduct of the Hopf monoid $\operatorname{scf}(\mathrm{U})$ takes the same form on the $\lambda$-basis as on the $\kappa$-basis.

Proposition 15. Let $I=S_{1} \sqcup S_{2}$ and $\ell \in \mathrm{L}[I]$. Then

$$
\Delta_{S_{1}, S_{2}}\left(\lambda_{X, \alpha}\right)= \begin{cases}\lambda_{\left.X\right|_{S_{1}},\left.\alpha\right|_{S_{1}}} \otimes \lambda_{\left.X\right|_{S_{2}},\left.\alpha\right|_{S_{2}}} & \text { if } S_{1} \text { is the union of some blocks of } X, \\ 0 & \text { otherwise. }\end{cases}
$$

Proof. Suppose first that $S_{1}$ is not the union of blocks of $X$. Then the same is true for any partition coarser than $X$, in particular for any partition $Y$ entering in (45). In view of (43), we then have $\Delta_{S_{1}, S_{2}}\left(\lambda_{X, \alpha}\right)=0$.

Otherwise, $X=\left.\left.X\right|_{S_{1}} \sqcup X\right|_{S_{2}}$ and $\alpha=\left.\left.\alpha\right|_{S_{1}} \sqcup \alpha\right|_{S_{2}}$. Among the arc diagrams $(Y, \beta)$ entering in (45), only those for which $Y=\left.\left.Y\right|_{S_{1}} \sqcup Y\right|_{S_{2}}$ contribute to the coproduct, in view of (43). These arc diagrams are of the form $Y=Y_{1} \sqcup Y_{2}$ and $\beta=\beta_{1} \sqcup \beta_{2}$, and by (40), we must have

$$
A\left(\left.X\right|_{S_{i}},\left.\ell\right|_{S_{i}}\right) \subseteq A\left(Y_{i},\left.\ell\right|_{S_{i}}\right) \quad \text { and }\left.\quad \beta_{i}\right|_{A\left(\left.X\right|_{S_{i}},\left.\ell\right|_{S_{i}}\right)}=\left.\alpha\right|_{S_{i}}, \quad i=1,2 .
$$

We then have

$$
\begin{aligned}
\Delta_{S_{1}, S_{2}}\left(\lambda_{X, \alpha}\right) & =\sum_{(X, \alpha) \leq(Y, \beta)} \Delta\left(\kappa_{Y, \beta}\right) \\
& =\sum_{\left(\left.X\right|_{S_{i}},\left.\alpha\right|_{S_{i}}\right) \leq\left(Y_{i}, \beta_{i}\right)} \kappa_{Y_{1}, \beta_{1}} \otimes \kappa_{Y_{2}, \beta_{2}}=\lambda_{\left.X\right|_{S_{1}},\left.\alpha\right|_{S_{1}}} \otimes \lambda_{\left.X\right|_{S_{2}},\left.\alpha\right|_{S_{2}}} .
\end{aligned}
$$

Remark 16. The relationship between the $\lambda$ - and $\kappa$-bases of $\operatorname{scf}(\mathrm{U})$ is somewhat reminiscent of that between the $p$ - and $m$-bases of $\Pi$ [Aguiar and Mahajan 2010, Equation (12.5)]. However, the latter involves a sum over all partitions coarser than a given one. For this reason, the morphism $\phi$ in (44), which relates the $m$-basis to the $\kappa$-basis, does not relate the $p$-basis to the $\lambda$-basis in the same manner.

5.3. Freeness of $\operatorname{scf}(\mathbf{U})$. Let $\boldsymbol{q}$ be a species such that $\boldsymbol{q}[\varnothing]=0$. A new species $\mathscr{T}(\boldsymbol{q})$ is defined by $\mathscr{T}(\boldsymbol{q})[\varnothing]=\mathbb{k}$ and, on a finite nonempty set $I$,

$$
\mathscr{T}(\boldsymbol{q})[I]=\bigoplus_{\substack{I=I_{1} \cup \cdots \cup I_{k} \\ k \geq 1, I_{j} \neq \varnothing}} \boldsymbol{q}\left[I_{1}\right] \otimes \cdots \otimes \boldsymbol{q}\left[I_{k}\right] .
$$

The sum is over all decompositions of $I$ into nonempty subsets. The number $k$ of subsets is therefore bounded above by $|I|$. 
The species $\mathscr{T}(\boldsymbol{q})$ is a connected monoid with product given by concatenation. To describe this in detail, let $I=S \sqcup T$ and choose decompositions $S=S_{1} \sqcup \cdots \sqcup S_{k}$ and $T=T_{1} \sqcup \cdots \sqcup T_{l}$ and elements $x_{i} \in \boldsymbol{q}\left[S_{i}\right], i=1, \ldots, k$, and $y_{j} \in \boldsymbol{q}\left[T_{j}\right], j=1, \ldots, l$. Write

$$
x=x_{1} \otimes \cdots \otimes x_{i} \in \mathscr{T}(\boldsymbol{q})[S] \quad \text { and } \quad y=y_{1} \otimes \cdots \otimes y_{j} \in \mathscr{T}(\boldsymbol{q})[T] .
$$

The product is

$$
\begin{aligned}
\mu_{S, T}(x \otimes y)=x_{1} \otimes \cdots \otimes & x_{i} \otimes y_{1} \otimes \cdots \otimes y_{j} \\
& \quad \in \boldsymbol{q}\left[S_{1}\right] \otimes \cdots \otimes \boldsymbol{q}\left[S_{k}\right] \otimes \boldsymbol{q}\left[T_{1}\right] \otimes \cdots \otimes \boldsymbol{q}\left[T_{l}\right] \subseteq \mathscr{T}(\boldsymbol{q})[I] .
\end{aligned}
$$

The monoid $\mathscr{T}(\boldsymbol{q})$ is free on the species $\boldsymbol{q}$ : a map of species $\boldsymbol{q} \rightarrow \boldsymbol{m}$ from $\boldsymbol{q}$ to a monoid $\boldsymbol{m}$ has a unique extension to a morphism of monoids $\mathscr{T}(\boldsymbol{q}) \rightarrow \boldsymbol{m}$.

The monoid $\mathscr{T}(\boldsymbol{q})$ may carry several coproducts that turn it into a connected Hopf monoid. The canonical structure is the one for which the elements of $\boldsymbol{q}$ are primitive. This means that

$$
\Delta_{S, T}(x)=0
$$

for every $x \in q[I]$ and every decomposition $I=S \sqcup T$ into nonempty subsets.

More details can be found in [Aguiar and Mahajan 2010, Sections 11.2.1-11.2.2].

Let $\mathrm{D}(I, \ell)$ denote the set of arc diagrams $(X, \alpha)$ for which $X$ is an atomic set partition of the linearly ordered set $(I, \ell)$. Let $\boldsymbol{d}(I, \ell)$ be the vector space with basis $\mathrm{D}(I, \ell)$. Define a species $\boldsymbol{d}$ by

$$
d[I]=\bigoplus_{\ell \in \mathrm{L}[I]} d(I, \ell)
$$

Consider the map of species $\boldsymbol{d} \rightarrow$ scf with components

$$
d[I] \rightarrow \operatorname{scf}(\mathrm{U})[I], \quad(X, \alpha) \mapsto \lambda_{X, \alpha} .
$$

The map sends the summand $\boldsymbol{d}(I, \ell)$ of $\boldsymbol{d}[I]$ to the summand $\operatorname{scf}(\mathrm{U}(I, \ell))$ of $\operatorname{scf}(\mathrm{U})[I]$. By freeness, it extends to a morphism of monoids

$$
\mathscr{T}(d) \rightarrow \operatorname{scf}(\mathrm{U}) .
$$

Proposition 17. The map $\mathscr{T}(\boldsymbol{d}) \rightarrow \operatorname{scf}(\mathrm{U})$ is an isomorphism of monoids. In particular, the monoid $\operatorname{scf}(\mathrm{U})$ is free.

Proof. Let $(X, \alpha)$ be an arbitrary arc diagram on $(I, \ell)$. Let $I_{1}, \ldots, I_{k}$ be the minimal $\ell$-segments of $I$, numbered from left to right, such that each $I_{j}$ is a union of blocks of $X$. Let $\ell_{j}=\left.\ell\right|_{I_{j}}, X_{j}=\left.X\right|_{I_{j}}$, and $\alpha_{j}=\left.\alpha\right|_{I_{j}}$. Then $X_{j}$ is an atomic partition of $\left(I_{j}, \ell_{j}\right)$,

$$
X_{1} \sqcup \cdots \sqcup X_{j}=X \quad \text { and } \quad \alpha_{1} \sqcup \cdots \sqcup \alpha_{j}=\alpha .
$$


By (46),

$$
\mu_{I_{1}, \ldots, I_{k}}\left(\lambda_{X_{1}, \alpha_{1}} \otimes \cdots \otimes \lambda_{X_{k}, \alpha_{k}}\right)=\lambda_{X, \alpha}
$$

Thus, the morphism $\mathscr{T}(\boldsymbol{d}) \rightarrow \operatorname{scf}(\mathrm{U})$ sends the basis element $\left(X_{1}, \alpha_{1}\right) \otimes \cdots \otimes$ $\left(X_{k}, \alpha_{k}\right)$ of $\boldsymbol{d}\left(I_{1}, \ell_{1}\right) \otimes \cdots \otimes \boldsymbol{d}\left(I_{k}, \ell_{k}\right)$ to the basis element $\lambda_{X, \alpha}$ of $\operatorname{scf}(\mathrm{U}(I, \ell))$ and is therefore an isomorphism.

We may state Proposition 17 by saying that the superclass functions $\lambda_{X, \alpha}$ freely generate the monoid $\operatorname{scf}(\mathrm{U})$ as $(X, \alpha)$ runs over all arc diagrams for which $X$ is an atomic set partition.

The generators, however, need not be primitive. For instance,
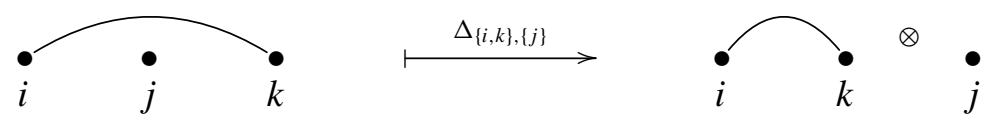

which is not 0 . Nevertheless, Proposition 23 allows us to conclude the following:

Corollary 18. Let $\mathbb{k}$ be a field of characteristic 0 . There exists an isomorphism of Hopf monoids $\operatorname{scf}(\mathrm{U}) \cong \mathscr{T}(\boldsymbol{d})$, where the latter is endowed with its canonical Hopf structure.

As discussed in the Appendix, an isomorphism may be constructed with the aid of the first Eulerian idempotent.

Let $\Pi_{a}(I, \ell)$ be the vector space with basis the set of atomic partitions on $(I, \ell)$. When the field of matrix entries is $\mathbb{F}_{2}$, arc diagrams reduce to atomic set partitions and $\boldsymbol{d}(I, \ell)$ identifies with $\boldsymbol{\Pi}_{a}(I, \ell)$. Combining Corollaries 12 and 18, we obtain an isomorphism of Hopf monoids

$$
\boldsymbol{L} \times \boldsymbol{\Pi} \cong \mathscr{T}\left(\boldsymbol{\Pi}_{a}\right),
$$

where

$$
\boldsymbol{\Pi}_{a}[I]=\bigoplus_{\ell \in \mathrm{L}[I]} \boldsymbol{\Pi}_{a}(I, \ell)
$$

5.4. A second basis for $\boldsymbol{G}$ and for $\mathbf{f}(\mathrm{U})$. Given two unitriangular matrices $U$ and $V \in \mathrm{U}(I, \ell)$, let us write $U \leq V$ if

$$
u_{i j}=v_{i j} \quad \text { whenever } u_{i j} \neq 0 \text {. }
$$

In other words, some zero entries in $U$ may be nonzero in $V$; the other entries are the same for both matrices.

We define a new basis $\left\{\lambda_{U}\right\}$ of $f(U(I, \ell))$ by

$$
\lambda_{U}=\sum_{U \leq V} \kappa_{V}
$$


Let $I=S_{1} \sqcup S_{2}, U \in \mathrm{U}(I, \ell)$, and $g_{i} \in \mathrm{U}\left(S_{i}, \ell_{i}\right), i=1,2$. It is easy to derive the following formulas from (29) and (30):

$$
\begin{gathered}
\mu_{S_{1}, S_{2}}\left(\lambda_{U_{1}} \otimes \lambda_{U_{2}}\right)=\lambda_{U_{1} \oplus U_{2}}, \\
\Delta_{S_{1}, S_{2}}\left(\lambda_{U}\right)= \begin{cases}\lambda_{U_{S_{1}}} \otimes \lambda_{U_{S_{2}}} & \text { if } U=U_{S_{1}} \oplus U_{S_{2}}, \\
0 & \text { otherwise. }\end{cases}
\end{gathered}
$$

(49) implies that $\mathbf{f}(\mathrm{U})$ is a free monoid with generators $\lambda_{U}$ indexed by unitriangular matrices $U$ for which the graph $g(U)$ is connected.

For completeness, one may define a new basis $\left\{p_{g}\right\}$ of $\boldsymbol{G}[I]$ by

$$
p_{g}=\sum_{g \subseteq h} m_{h}
$$

The sum is over all simple graphs $h$ with vertex set $I$ and with the same or more edges than $g$. Let $I=S_{1} \sqcup S_{2}, g \in \mathrm{G}[I]$, and $g_{i} \in \mathrm{G}\left[S_{i}\right], i=1$, 2. From (9) and (10), one obtains

$$
\begin{gathered}
\mu_{S_{1}, S_{2}}\left(p_{g_{1}} \otimes p_{g_{2}}\right)=p_{g_{1} \sqcup g_{2}}, \\
\Delta_{S_{1}, S_{2}}\left(p_{g}\right)= \begin{cases}p_{\left.g\right|_{S_{1}}} \otimes p_{\left.g\right|_{S_{2}}} & \text { if no edge of } g \text { connects } S_{1} \text { to } S_{2}, \\
0 & \text { otherwise. }\end{cases}
\end{gathered}
$$

Equation (52) implies that $\boldsymbol{G}$ is the free commutative monoid on the species of connected graphs. From (44), we deduce that the morphism $\phi$ of Proposition 8 takes the following form on these bases:

$$
\phi\left(\ell \otimes p_{g}\right)=\sum_{\substack{U \in \mathrm{U}(I, \ell) \\ g(U)=g}} \lambda_{U} .
$$

\section{Applications}

We conclude with some applications and remarks regarding past and future work.

6.1. Counting conjugacy classes. Let $k_{n}(q)$ be the number of conjugacy classes of the group of unitriangular matrices of size $n$ with entries in the field with $q$ elements. Higman's conjecture states that, for fixed $n, k_{n}$ is a polynomial function of $q$. Much effort has been devoted to the precise determination of these numbers or their asymptotic behavior [Goodwin 2006; Goodwin and Röhrle 2009; Higman 1960; Robinson 1998; Vera-López and Arregi 2003; Vera-López et al. 2008].

We fix $q$ and let $n$ vary. It turns out that the existence of a Hopf monoid structure on class functions imposes certain linear conditions on the sequence $k_{n}(q)$, as we explain next. 
Given a finite-dimensional Hopf monoid $\boldsymbol{h}$, consider the generating function

$$
\mathrm{T}_{\boldsymbol{h}}(x)=\sum_{n \geq 0} \operatorname{dim}_{\mathbb{k}}\left(\boldsymbol{h}[n]_{\mathrm{S}_{n}}\right) x^{n} .
$$

Here $[n]$ denotes the set $\{1,2, \ldots, n\}$ and $\boldsymbol{h}[n]_{\mathrm{S}_{n}}$ is the (quotient) space of coinvariants for the action of the symmetric group (afforded by the species structure of $\boldsymbol{h}$ ).

For example, since

$$
(\boldsymbol{L} \times \boldsymbol{\Pi})[n]_{\mathrm{S}_{n}}=(\boldsymbol{L}[n] \otimes \Pi[n])_{\mathrm{S}_{n}} \cong \Pi[n],
$$

we have

$$
\mathrm{T}_{\boldsymbol{L} \times \boldsymbol{\Pi}}(x)=\sum_{n \geq 0} B_{n} x^{n}
$$

where $B_{n}$ is the $n$-th Bell number, the number of partitions of the set [ $\left.n\right]$.

On the other hand, from (48),

$$
\mathrm{T}_{\boldsymbol{L} \times \boldsymbol{\Pi}}(x)=\mathrm{T}_{\mathscr{T}\left(\Pi_{a}\right)}(x) .
$$

It is a general fact that, for a species $\boldsymbol{q}$ with $\boldsymbol{q}[\varnothing]=0$,

$$
\mathrm{T}_{\mathscr{T}(\boldsymbol{q})}(x)=\frac{1}{1-\mathrm{T}_{\boldsymbol{q}}(x)} .
$$

(This follows from [Bergeron_F et al. 1998, Theorem 2.b, Section 1.4] for instance). Therefore,

$$
\mathrm{T}_{\boldsymbol{L} \times \boldsymbol{\Pi}}(x)=\frac{1}{1-\sum_{n \geq 1} A_{n} x^{n}},
$$

where $A_{n}$ is the number of atomic partitions of the linearly ordered set $[n]$.

From (55) and (56), we deduce that

$$
\sum_{n \geq 0} B_{n} x^{n}=\frac{1}{1-\sum_{n \geq 1} A_{n} x^{n}},
$$

a fact known from [Bergeron and Zabrocki 2009].

Consider now the injections

$$
\operatorname{scf}(\mathrm{U}) \hookrightarrow \operatorname{cf}(\mathrm{U}) \quad \text { and } \quad L \times \Pi \hookrightarrow \operatorname{scf}(\mathrm{U})
$$

Both are morphisms of Hopf monoids (Propositions 10 and 11). Lagrange's theorem for Hopf monoids implies in this situation that both quotients

$$
\frac{\mathrm{T}_{\mathbf{c f}(\mathrm{U})}(x)}{\mathrm{T}_{\operatorname{scf}(\mathrm{U})}(x)} \text { and } \frac{\mathrm{T}_{\operatorname{scf}_{(\mathrm{U})}}(x)}{\mathrm{T}_{L \times \Pi}(x)}
$$


belong to $\mathbb{N}[[x]$, that is, have nonnegative (integer) coefficients [Aguiar and Lauve 2012, Corollary 13]. In particular,

$$
\frac{\mathrm{T}_{\mathbf{c f}(\mathrm{U})}(x)}{\mathrm{T}_{\boldsymbol{L} \times \boldsymbol{\Pi}}(x)} \in \mathbb{N} \llbracket x \rrbracket
$$

as well.

We have

$$
\operatorname{cf}(\mathrm{U})[n]_{\mathrm{S}_{n}}=\left(\bigoplus_{\ell \in \mathrm{L}[n]} \operatorname{cf}(\mathrm{U}([n], \ell))\right)_{\mathrm{S}_{n}} \cong \operatorname{cf}(\mathrm{U}([n])) .
$$

Therefore,

$$
\mathrm{T}_{\mathbf{c f}(\mathrm{U})}(x)=\sum_{n \geq 0} k_{n}(q) x^{n} .
$$

By combining the above, we deduce

$$
\left.\left(\sum_{n \geq 0} k_{n}(q) x^{n}\right)\left(1-\sum_{n \geq 1} A_{n} x^{n}\right) \in \mathbb{N}[x]\right],
$$

whence the following result:

Corollary 19. The following linear inequalities are satisfied for every $n \in \mathbb{N}$ and every prime power $q$ :

$$
k_{n}(q) \geq \sum_{i=0}^{n-1} A_{n-i} k_{i}(q) .
$$

For instance, for $n=8$, the inequality is

$$
k_{6}(q) \geq 92+22 k_{1}(q)+6 k_{2}(q)+2 k_{3}(q)+k_{4}(q)+k_{5}(q) .
$$

Inequality (57) is stronger than merely stating that there are more conjugacy classes than superclasses. For instance, for $q=2$ and $n=6$, the right-hand side of the inequality is 213 (provided we use the correct values for $k_{i}(2)$ for $i \leq 5$ ) while there are only $B_{6}=203$ superclasses. The first few values of the sequence $k_{n}(2)$ appear in [OEIS Foundation 2010] as A007976; in particular, $k_{6}(2)=275$.

The numbers $k_{n}(q)$ are known for $n \leq 13$ from work of Vera-López and Arregi [1992; 1995; 2003]; see also [Vera-López et al. 2008]. (There is an incorrect sign in the value given for $k_{7}(q)$ in [Vera-López and Arregi 1995, page 923]: the lowest term should be $-7 q$.)

We may derive additional information on these numbers from the injective morphism of Hopf monoids (Proposition 6)

$$
\operatorname{cf}(\mathrm{U}) \hookrightarrow f(U) .
$$


Define a sequence of integers $c_{n}(q), n \geq 1$, by means of

$$
\sum_{n \geq 0} k_{n}(q) x^{n}=\frac{1}{1-\sum_{n \geq 1} c_{n}(q) x^{n}} .
$$

Arguing as above, we obtain the following result:

Corollary 20. The following linear inequalities are satisfied for every $n \in \mathbb{N}$ and every prime power $q$ :

$$
q^{\left(\begin{array}{c}
n \\
2
\end{array}\right)} \geq \sum_{i=1}^{n} q^{\left(\begin{array}{c}
n-i \\
2
\end{array}\right)} c_{i}(q) .
$$

Through (58), these inequalities impose further constraints on the numbers $k_{n}(q)$. The first few values of the sequence $c_{n}(q)$ are as follows with $t=q-1$ :

$$
\begin{aligned}
& c_{1}(q)=1, \\
& c_{2}(q)=t, \\
& c_{3}(q)=t^{2}+t \\
& c_{4}(q)=2 t^{3}+4 t^{2}+t, \\
& c_{5}(q)=5 t^{4}+14 t^{3}+9 t^{2}+t, \\
& c_{6}(q)=t^{6}+18 t^{5}+55 t^{4}+54 t^{3}+16 t^{2}+t .
\end{aligned}
$$

Conjecture 21. There exist polynomials $p_{n}(t) \in \mathbb{N}[t]$ such that $c_{n}(q)=p_{n}(q-1)$ for every prime power $q$ and every $n \geq 1$.

Using the formulas given by Vera-López et al. [2008, Corollaries 10-11] for computing $k_{n}(q)$, we have verified the conjecture for $n \leq 13$.

Polynomiality of $k_{n}(q)$ is equivalent to that of $c_{n}(q)$. On the other hand, the nonnegativity of $c_{n}$ as a polynomial of $t$ implies that of $k_{n}$ but not conversely. Thus, Conjecture 21 is a strong form of Higman's.

It is possible to show, using the methods of [Aguiar and Mahajan 2012], that the monoid $\mathbf{c f}(\mathrm{U})$ is free. This implies that the integers $c_{n}(q)$ are nonnegative for every $n \geq 1$ and prime power $q$.

6.2. From Hopf monoids to Hopf algebras. It is possible to associate a number of graded Hopf algebras to a given Hopf monoid $\boldsymbol{h}$. This is the subject of [Aguiar and Mahajan 2010, Part III]. In particular, there are two graded Hopf algebras $\mathscr{K}(\boldsymbol{h})$ and $\overline{\mathscr{K}}(\boldsymbol{h})$ related by a canonical surjective morphism

$$
\mathscr{K}(\boldsymbol{h}) \rightarrow \overline{\mathscr{K}}(\boldsymbol{h}) .
$$


The underlying spaces of these Hopf algebras are

$$
\mathscr{K}(\boldsymbol{h})=\bigoplus_{n \geq 0} \boldsymbol{h}[n] \quad \text { and } \quad \overline{\mathscr{K}}(\boldsymbol{h})=\bigoplus_{n \geq 0} \boldsymbol{h}[n]_{\mathrm{S}_{n}},
$$

where $\boldsymbol{h}[n]_{\mathrm{S}_{n}}$ is as in (54). The product and coproduct of these Hopf algebras is built from those of the Hopf monoid $\boldsymbol{h}$ together with certain canonical transformations. The latter involve certain combinatorial procedures known as shifting and standardization. For more details, we refer to [Aguiar and Mahajan 2010, Chapter 15].

For example, one has that

$$
\overline{\mathscr{K}}(\boldsymbol{L})=\mathbb{k}[x]
$$

is the polynomial algebra on one primitive generator while $\mathscr{K}(\boldsymbol{L})$ is the Hopf algebra introduced by Patras and Reutenauer [2004].

According to [Aguiar and Mahajan 2010, Section 17.4], $\overline{\mathscr{K}}(\boldsymbol{\Pi})$ is the ubiquitous Hopf algebra of symmetric functions while $\mathscr{K}(\boldsymbol{\Pi})$ is the Hopf algebra of symmetric functions in noncommuting variables, an object studied in various references including [Aguiar and Mahajan 2006, Section 6.2; Bergeron et al. 2006; Bergeron and Zabrocki 2009; Rosas and Sagan 2006].

For any Hopf monoid $\boldsymbol{h}$, one has [Aguiar and Mahajan 2010, Theorem 15.13]

$$
\overline{\mathscr{K}}(\boldsymbol{L} \times \boldsymbol{h}) \cong \mathscr{K}(\boldsymbol{h}) .
$$

Combining with Corollary 12, we obtain that, when the field of coefficients is $\mathbb{F}_{2}$,

$$
\overline{\mathscr{K}}(\operatorname{scf}(\mathrm{U})) \cong \overline{\mathscr{K}}(\boldsymbol{L} \times \boldsymbol{\Pi}) \cong \mathscr{K}(\boldsymbol{\Pi}) .
$$

In other words, the Hopf algebra constructed from superclass functions on unitriangular matrices (with entries in $\mathbb{F}_{2}$ ) via the functor $\overline{\mathscr{K}}$ is isomorphic to the Hopf algebra of symmetric functions in noncommuting variables. This is the main result of [Aguiar et al. 2012].

The freeness of the Hopf algebra $\mathscr{K}(\Pi)$, a fact known from [Harčenko 1978; Wolf 1936], is a consequence of Proposition 17.

We mention that one may arrive at Corollary 19 by employing the Hopf algebra $\overline{\mathscr{K}}(\mathbf{c f}(\mathrm{U}))$ (rather than the Hopf monoid $\operatorname{cf}(\mathrm{U})$ ) and appealing to Lagrange's theorem for graded connected Hopf algebras.

6.3. Supercharacters and beyond. The notion of superclass on a unitriangular group comes with a companion notion of supercharacter and a full-fledged theory relating them. This is due to the pioneering work of André [1995a; 1995b] and later Yan [2001]. Much of this theory extends to algebra groups [André 1999; Diaconis and Isaacs 2008; Diaconis and Thiem 2009]. More recently, a connection with classical work on Schur rings has been understood [Hendrickson 2010]. 
In regards to the object of present interest, the Hopf monoid $\operatorname{scf}(\mathrm{U})$, this implies the existence of a second canonical linear basis consisting of supercharacters. The work of André and Yan provides a character formula, which yields the change of basis between superclass functions and supercharacters. We plan to study the Hopf monoid structure of $\operatorname{scf}(\mathrm{U})$ on the supercharacter basis in future work.

\section{Appendix: On free Hopf algebras and Hopf monoids}

A free algebra may carry several Hopf algebra structures. It always carries a canonical one in which the generators are primitive. It turns out that under certain conditions, any Hopf structure on a free algebra is isomorphic to the canonical one. We provide such a result below. An analogous result holds for Hopf monoids in vector species. This is applied in the paper in Section 5.

We assume that the base field $\mathbb{k}$ is of characteristic 0 .

We employ the first Eulerian idempotent [Gerstenhaber and Schack 1991; Loday 1998, Section 4.5.2; Reutenauer 1993, Section 8.4]. For any connected Hopf algebra $H$, the identity map id : $H \rightarrow H$ is locally unipotent with respect to the convolution product of $\operatorname{End}(H)$. Therefore,

$$
\boldsymbol{e}:=\log (\mathrm{id})=\sum_{k \geq 1} \frac{(-1)^{k+1}}{k}(\mathrm{id}-\iota \epsilon)^{* k}
$$

is a well-defined linear endomorphism of $H$. Here

$$
\iota: \mathbb{k} \rightarrow H \quad \text { and } \quad \epsilon: H \rightarrow \mathbb{k}
$$

denote the unit and counit maps of $H$, respectively, and the powers are with respect to the convolution product. It is an important fact that if $H$ is in addition cocommutative, then $\boldsymbol{e}(x)$ is a primitive element of $H$ for any $x \in H$. In fact, the operator $\boldsymbol{e}$ is in this case a projection onto the space of primitive elements [Patras 1994; Schmitt 1994, pages 314-318].

Let $T(V)$ denote the free algebra on a vector space $V$ :

$$
T(V)=\bigoplus_{n \geq 0} V^{\otimes n}
$$

The product is concatenation of tensors. We say in this case that $V$ freely generates.

The unique morphisms of algebras

$$
\Delta: T(V) \rightarrow T(V) \otimes T(V) \quad \text { and } \quad \epsilon: T(V) \rightarrow \mathbb{k}
$$

given for all $v \in V$ by

$$
\Delta(v)=1 \otimes v+v \otimes 1 \quad \text { and } \quad \epsilon(v)=0
$$


turn $T(V)$ into a connected, cocommutative Hopf algebra. This is the canonical Hopf structure on $T(V)$.

Proposition 22. Let $\mathbb{k}$ be a field of characteristic 0 . Let $H$ be a connected cocommutative Hopf algebra over $\mathbb{k}$. Suppose $H \cong T(W)$ as algebras in such a way that the image of $W$ lies in the kernel of the $\epsilon$. Then there exists a (possibly different) isomorphism of Hopf algebras $H \cong T(W)$, where the latter is endowed with its canonical Hopf structure.

Proof. We may assume $H=T(W)$ as algebras for some subspace $W$ of $\operatorname{ker}(\epsilon)$. Since $H$ is connected and $\mathbb{k}$ is of characteristic 0 , the Eulerian idempotent $\boldsymbol{e}$ is defined. Let $V=\boldsymbol{e}(W)$. We show below that $V \cong W$ and that $V$ freely generates $H$. Since $H$ is cocommutative, $V$ consists of primitive elements, and therefore, $H \cong T(V)$ as Hopf algebras. This completes the proof.

Let

$$
H_{+}=\bigoplus_{n \geq 1} W^{\otimes n}
$$

Since $\epsilon$ is a morphism of algebras, $H_{+} \subseteq \operatorname{ker}(\epsilon)$, and since both spaces are of codimension 1, they must agree: $H_{+}=\operatorname{ker}(\epsilon)$.

Define $\Delta_{+}: H_{+} \rightarrow H_{+} \otimes H_{+}$by

$$
\Delta_{+}(x)=\Delta(x)-1 \otimes x-x \otimes 1 .
$$

By counitality,

$$
(\epsilon \otimes \mathrm{id}) \Delta_{+}=0=(\mathrm{id} \otimes \epsilon) \Delta_{+} .
$$

Therefore, $\Delta_{+}\left(H_{+}\right) \subseteq \operatorname{ker}(\epsilon) \otimes \operatorname{ker}(\epsilon)=H_{+} \otimes H_{+}$, and hence,

$$
\Delta_{+}^{(k-1)}\left(H_{+}\right) \subseteq H_{+}^{\otimes k}
$$

for all $k \geq 1$. In addition, since $H=T(W)$ as algebras,

$$
\mu^{(k-1)}\left(H_{+}^{\otimes k}\right) \subseteq \sum_{n \geq 2} W^{\otimes n}
$$

for all $k \geq 2$.

Take $w \in W$. Then

$$
\begin{aligned}
\boldsymbol{e}(w) & =\sum_{k \geq 1} \frac{(-1)^{k+1}}{k}(\mathrm{id}-\iota \epsilon)^{* k}(w)=w+\sum_{k \geq 2} \frac{(-1)^{k+1}}{k} \mu^{(k-1)} \Delta_{+}^{(k-1)}(w) \\
& \equiv w+\sum_{n \geq 2} W^{\otimes n} .
\end{aligned}
$$

By triangularity, $\boldsymbol{e}: W \rightarrow V$ is invertible, and hence, $V$ generates $H$. 
Now take $w_{1}, w_{2} \in W$. It follows from the above that

$$
\boldsymbol{e}\left(w_{1}\right) \boldsymbol{e}\left(w_{2}\right) \equiv w_{1} w_{2}+\sum_{n \geq 3} W^{\otimes n},
$$

and a similar triangular relation holds for higher products. Hence, $V$ generates $H$ freely.

The Eulerian idempotent is defined for connected Hopf monoids in species by the same formula as (60). Let $\boldsymbol{p}$ be a species such that $\boldsymbol{p}[\varnothing]=0$. The free monoid $\mathscr{T}(\boldsymbol{p})$ and its canonical Hopf structure is discussed in [Aguiar and Mahajan 2010, Section 11.2]. The arguments in Proposition 22 may easily be adapted to this setting to yield the following result:

Proposition 23. Let $\mathbb{k}$ be a field of characteristic 0 . Let $\boldsymbol{h}$ be a connected cocommutative Hopf monoid in vector species over $\mathbb{k}$. Suppose $\boldsymbol{h} \cong \mathcal{T}(\boldsymbol{p})$ as monoids for some species $\boldsymbol{p}$ such that $\boldsymbol{p}[\varnothing]=0$. Then there exists a (possibly different) isomorphism of Hopf monoids $\boldsymbol{h} \cong \mathscr{T}(\boldsymbol{p})$, where the latter is endowed with its canonical Hopf structure.

\section{Acknowledgements}

We thank the referee and Franco Saliola for useful comments and suggestions.

\section{References}

[Aguiar and Lauve 2012] M. Aguiar and A. Lauve, "Lagrange's theorem for Hopf monoids in species", preprint, 2012. arXiv 1105.5572v3

[Aguiar and Mahajan 2006] M. Aguiar and S. Mahajan, Coxeter groups and Hopf algebras, Fields Institute Monographs 23, American Mathematical Society, Providence, RI, 2006. MR 2008d:20072 Zbl 1106.16039

[Aguiar and Mahajan 2010] M. Aguiar and S. Mahajan, Monoidal functors, species and Hopf algebras, CRM Monograph Series 29, American Mathematical Society, Providence, RI, 2010. MR 2012g: 18009 Zbl 1209.18002

[Aguiar and Mahajan 2012] M. Aguiar and S. Mahajan, "On the Hadamard product of Hopf monoids", preprint, 2012. arXiv 1209.1363

[Aguiar et al. 2012] M. Aguiar, C. André, C. Benedetti, N. Bergeron, Z. Chen, P. Diaconis, A. Hendrickson, S. Hsiao, I. M. Isaacs, A. Jedwab, K. Johnson, G. Karaali, A. Lauve, T. Le, S. Lewis, H. Li, K. Magaard, E. Marberg, J.-C. Novelli, A. Pang, F. Saliola, L. Tevlin, J.-Y. Thibon, N. Thiem, V. Venkateswaran, C. R. Vinroot, N. Yan, and M. Zabrocki, "Supercharacters, symmetric functions in noncommuting variables, and related Hopf algebras", Adv. Math. 229:4 (2012), 2310-2337. MR 2880223 Zbl 1237.05208

[André 1995a] C. A. M. André, "Basic characters of the unitriangular group", J. Algebra 175:1 (1995), 287-319. MR 96h:20081a Zbl 0835.20052

[André 1995b] C. A. M. André, "Basic sums of coadjoint orbits of the unitriangular group", J. Algebra 176:3 (1995), 959-1000. MR 96h:20081b Zbl 0837.20050 
[André 1999] C. A. M. André, "Irreducible characters of finite algebra groups", pp. 65-80 in Matrices and group representations (Coimbra, 1998), Textos Mat. Sér. B 19, Universidade de Coimbra, Coimbra, 1999. MR 2001g:20009 Zbl 0972.20005

[Bergeron_F et al. 1998] F. Bergeron, G. Labelle, and P. Leroux, Combinatorial species and tree-like structures, Encyclopedia of Mathematics and its Applications 67, Cambridge University Press, 1998. MR 2000a:05008 Zbl 0888.05001

[Bergeron and Zabrocki 2009] N. Bergeron and M. Zabrocki, "The Hopf algebras of symmetric functions and quasi-symmetric functions in non-commutative variables are free and co-free", $J$. Algebra Appl. 8:4 (2009), 581-600. MR 2011a:05372 Zbl 1188.16030

[Bergeron et al. 2006] N. Bergeron, C. Hohlweg, M. Rosas, and M. Zabrocki, "Grothendieck bialgebras, partition lattices, and symmetric functions in noncommutative variables", Electron. J. Combin. 13:1 (2006), R75. MR 2007e:05176 Zbl 1098.05079

[Diaconis and Isaacs 2008] P. Diaconis and I. M. Isaacs, "Supercharacters and superclasses for algebra groups”, Trans. Amer. Math. Soc. 360:5 (2008), 2359-2392. MR 2009c:20012 Zbl 1137.20008

[Diaconis and Thiem 2009] P. Diaconis and N. Thiem, "Supercharacter formulas for pattern groups", Trans. Amer. Math. Soc. 361:7 (2009), 3501-3533. MR 2010g:20013 Zbl 1205.20006

[Gerstenhaber and Schack 1991] M. Gerstenhaber and S. D. Schack, "The shuffle bialgebra and the cohomology of commutative algebras”, J. Pure Appl. Algebra 70:3 (1991), 263-272. MR 92e:13008 Zbl 0728.13003

[Goodwin 2006] S. M. Goodwin, "On the conjugacy classes in maximal unipotent subgroups of simple algebraic groups”, Transform. Groups 11:1 (2006), 51-76. MR 2006k:20096 Zbl 1118.20041

[Goodwin and Röhrle 2009] S. M. Goodwin and G. Röhrle, "Calculating conjugacy classes in Sylow p-subgroups of finite Chevalley groups", J. Algebra 321:11 (2009), 3321-3334. MR 2010g:20083 Zbl 1210.20044

[Harčenko 1978] V. K. Harčenko, "Algebras of invariants of free algebras", Algebra i Logika 17:4 (1978), 478-487. In Russian; translated in Algebra and Logic 17:4 (1978), 316-321. MR 80e:16003

[Hendrickson 2010] A. O. F. Hendrickson, "Supercharacter theories and Schur rings", preprint, 2010. arXiv 1006.1363v1

[Higman 1960] G. Higman, "Enumerating p-groups, I: Inequalities", Proc. London Math. Soc. (3) 10 (1960), 24-30. MR 22 \#4779 Zbl 093.02603

[Isaacs 1995] I. M. Isaacs, "Characters of groups associated with finite algebras", J. Algebra 177:3 (1995), 708-730. MR 96k:20011 Zbl 0839.20010

[Kirillov 1995] A. A. Kirillov, "Variations on the triangular theme", pp. 43-73 in Lie groups and Lie algebras: E. B. Dynkin's seminar, edited by S. G. Gindikin and E. B. Vinberg, Amer. Math. Soc. Transl. Ser. 2 169, American Mathematical Society, Providence, RI, 1995. MR 97a:20072 Zbl 0840.22015

[Loday 1998] J.-L. Loday, Cyclic homology, 2nd ed., Grundlehren Math. Wiss. 301, Springer, Berlin, 1998. MR 98h:16014 Zbl 0885.18007

[OEIS Foundation 2010] OEIS Foundation, "The on-line encyclopedia of integer sequences", 2010, http://oeis.org.

[Patras 1994] F. Patras, “L'algèbre des descentes d'une bigèbre graduée”, J. Algebra 170:2 (1994), 547-566. MR 96a:16043 Zbl 0819.16033

[Patras and Reutenauer 2004] F. Patras and C. Reutenauer, "On descent algebras and twisted bialgebras”, Mosc. Math. J. 4:1 (2004), 199-216. MR 2005e:16067 Zbl 1103.16026 
[Reutenauer 1993] C. Reutenauer, Free Lie algebras, London Math. Soc. Monogr. (N.S.) 7, Oxford University Press, New York, 1993. MR 94j:17002 Zbl 0798.17001

[Robinson 1998] G. R. Robinson, "Counting conjugacy classes of unitriangular groups associated to finite-dimensional algebras", J. Group Theory 1:3 (1998), 271-274. MR 99h:14025 Zbl 0926. 20031

[Rosas and Sagan 2006] M. H. Rosas and B. E. Sagan, "Symmetric functions in noncommuting variables”, Trans. Amer. Math. Soc. 358:1 (2006), 215-232. MR 2006f:05184 Zbl 1071.05073

[Schmitt 1994] W. R. Schmitt, "Incidence Hopf algebras”, J. Pure Appl. Algebra 96:3 (1994), 299330. MR 95m:16033 Zbl 0808.05101

[Vera-López and Arregi 1992] A. Vera-López and J. M. Arregi, "Conjugacy classes in Sylow psubgroups of GL(n,q)”, J. Algebra 152:1 (1992), 1-19. MR 94b:20048 Zbl 0777.20015

[Vera-López and Arregi 1995] A. Vera-López and J. M. Arregi, "Some algorithms for the calculation of conjugacy classes in the Sylow p-subgroups of GL(n,q)", J. Algebra 177:3 (1995), 899-925. MR 96j:20029 Zbl 0839.20061

[Vera-López and Arregi 2003] A. Vera-López and J. M. Arregi, "Conjugacy classes in unitriangular matrices”, Linear Algebra Appl. 370 (2003), 85-124. MR 2004i:20091 Zbl 1045.20045

[Vera-López et al. 2008] A. Vera-López, J. M. Arregi, L. Ormaetxea, and F. J. Vera-López, “The exact number of conjugacy classes of the Sylow $p$-subgroups of $\operatorname{GL}(n, q)$ modulo $(q-1){ }^{13}$ ", Linear Algebra Appl. 429:2-3 (2008), 617-624. MR 2009d:20117 Zbl 1141.20008

[Wolf 1936] M. C. Wolf, "Symmetric functions of non-commutative elements", Duke Math. J. 2:4 (1936), 626-637. MR 1545953 Zbl 0016.00501

[Yan 2001] N. Yan, Representation theory of the finite unipotent linear groups, Ph.D. thesis, University of Pennsylvania, 2001, http://repository.upenn.edu/dissertations/AAI3015396/. MR 2702153

Communicated by Ravi Vakil

Received 2012-04-10 Revised 2012-08-08 Accepted 2012-10-07

maguiar@math.tamu.edu Department of Mathematics, Texas A\&M University, College Station, TX, 77843, United States http://www.math.tamu.edu/ maguiar

bergeron@mathstat.yorku.ca Department of Mathematics and Statistics, York University, Toronto, ON, M3J 1P3, Canada http://www.math.yorku.ca/bergeron

thiem@colorado.edu

Department of Mathematics, University of Colorado, Boulder, CO, 80309, United States http://math.colorado.edu/ thiemn 


\section{Algebra \& Number Theory}

msp.org/ant

\section{EDITORS}

MANAGING EDITOR

Bjorn Poonen

Massachusetts Institute of Technology

Cambridge, USA

\author{
EDITORIAL BOARD CHAIR \\ David Eisenbud \\ University of California \\ Berkeley, USA
}

\section{BOARD OF EDITORS}

Georgia Benkart

Dave Benson

Richard E. Borcherds

John H. Coates

J-L. Colliot-Thélène

Brian D. Conrad

Hélène Esnault

Hubert Flenner

Edward Frenkel

Andrew Granville

Joseph Gubeladze

Roger Heath-Brown

Ehud Hrushovski

Craig Huneke

Mikhail Kapranov

Yujiro Kawamata

János Kollár

Yuri Manin

Barry Mazur

Philippe Michel
University of Wisconsin, Madison, USA

University of Aberdeen, Scotland

University of California, Berkeley, USA

University of Cambridge, UK

CNRS, Université Paris-Sud, France

University of Michigan, USA

Freie Universität Berlin, Germany

Ruhr-Universität, Germany

University of California, Berkeley, USA

Université de Montréal, Canada

San Francisco State University, USA

Oxford University, UK

Hebrew University, Israel

University of Virginia, USA

Yale University, USA

University of Tokyo, Japan

Princeton University, USA

Northwestern University, USA

Harvard University, USA

École Polytechnique Fédérale de Lausanne
Susan Montgomery

Shigefumi Mori

Raman Parimala

Jonathan Pila

Victor Reiner

Karl Rubin

Peter Sarnak

Joseph H. Silverman

Michael Singer

Vasudevan Srinivas

J. Toby Stafford

Bernd Sturmfels

Richard Taylor

Ravi Vakil

Michel van den Bergh

Marie-France Vignéras

Kei-Ichi Watanabe

Efim Zelmanov

Shou-Wu Zhang
University of Southern California, USA

RIMS, Kyoto University, Japan

Emory University, USA

University of Oxford, UK

University of Minnesota, USA

University of California, Irvine, USA

Princeton University, USA

Brown University, USA

North Carolina State University, USA

Tata Inst. of Fund. Research, India

University of Michigan, USA

University of California, Berkeley, USA

Harvard University, USA

Stanford University, USA

Hasselt University, Belgium

Université Paris VII, France

Nihon University, Japan

University of California, San Diego, USA

Princeton University, USA

PRODUCTION

production@msp.org

Silvio Levy, Scientific Editor

See inside back cover or msp.org/ant for submission instructions.

The subscription price for 2013 is US $\$ 200 /$ year for the electronic version, and $\$ 350 /$ year $(+\$ 40$, if shipping outside the US) for print and electronic. Subscriptions, requests for back issues and changes of subscribers address should be sent to MSP.

Algebra \& Number Theory (ISSN 1944-7833 electronic, 1937-0652 printed) at Mathematical Sciences Publishers, 798 Evans Hall \#3840, c/o University of California, Berkeley, CA 94720-3840 is published continuously online. Periodical rate postage paid at Berkeley, CA 94704, and additional mailing offices.

ANT peer review and production are managed by EditFLOW ${ }^{\circledR}$ from Mathematical Sciences Publishers.

\section{PUBLISHED BY}

- mathematical sciences publishers

nonprofit scientific publishing

http://msp.org/

(C) 2013 Mathematical Sciences Publishers 


\section{Algebra \& Number Theory}

$\begin{array}{lll}\text { Volume } 7 & \text { No. } 7 \quad 2013\end{array}$

Weil representation and transfer factor

TERUJI THOMAS

Analytic families of finite-slope Selmer groups

1571

JONATHAN POTTHARST

Multiplicative excellent families of elliptic surfaces of type $E_{7}$ or $E_{8}$

ABHINAV KUMAR and TETSUJI SHIODA

Cohomological invariants of algebraic tori

SAM Blinstein and AleXANDer MerkurJeV

On abstract representations of the groups of rational points of algebraic groups and their 1685 deformations

IGOR A. RAPINCHUK

Betti diagrams from graphs

AlEXANDER ENGSTRÖM and MATTHEW T. STAMPS

Hopf monoids from class functions on unitriangular matrices

Marcelo Aguiar, NANTEl Bergeron and Nathaniel Thiem 